\title{
Long-time self-diffusion of charged colloidal particles: electrokinetic and hydrodynamic interaction effects
}

\author{
Mathieu G. McPhie and Gerhard Nägele \\ Institut für Festkörperforschung, Teilinstitut Weiche Materie, \\ Forschungszentrum Jülich, D-52425 Jülich, Germany*
}

(Dated: June 6, 2007)

\begin{abstract}
The authors analyze the long-time self-diffusion of charge-stabilized colloidal macroions in nondilute suspensions using a mode-coupling scheme developed for multi-component suspensions of interacting Brownian spheres. In this scheme, all ionic species, including counterions and electrolyte ions, are treated on an equal footing as charged hard spheres undergoing overdamped Brownian motion. Hydrodynamic interactions between all ions are accounted for on the far-field level. We show that the influence on the colloidal long-time self-diffusion coefficient arising from the relaxation of the microionic atmosphere surrounding the colloids, the so-called electrolyte friction effect, is usually insignificant in comparison with the friction contributions arising from direct and hydrodynamic interactions between the colloidal particles. This finding is true even for small colloid concentrations unless the mobility difference between colloidal particles and microions is not large. Furthermore, we observe an interesting non-monotonic density dependence of the colloidal long-time self-diffusion coefficient in suspensions with low amount of added salt. We show that this unusual density dependence is due to colloid-colloid hydrodynamic interactions.
\end{abstract}

\footnotetext{
*Electronic address: g. naegele@fz-juelich.de
} 


\section{INTRODUCTION}

The present work is concerned with the calculation of the long-time self-diffusion coefficient of charged colloidal spheres immersed in an electrolyte solution.

In colloidal self-diffusion, one considers the motion of a tagged colloidal particle relative to the other ones, and relative to the small electrolyte ions which form diffuse electric double layers around each macroion. In addition to the bare solvent friction, the charged colloidal particle experiences an electro-steric relaxation force which appears in response to the asymmetry in the surrounding electrolyte atmosphere, and in the distribution of neighboring macroions produced by its motion. This asymmetry is due to the finite mobilities of the electrolyte ions and the macroions which cannot respond instantaneously to the motion of the central macroion. The electro-steric friction contribution to the colloidal long-time self-diffusion coefficient, $D_{c}^{L}$, caused by the electrolyte ion kinetics is commonly referred to as the micro-ionic relaxation effect. The additional electro-steric friction on a colloidal sphere owing to the dynamic response of surrounding colloid particles is referred to instead as the electro-steric memory or caging effect. In general, $D_{c}^{L}$ is dominated by the electro-steric caging by neighboring macroions, since colloidal particles are much larger and slower, and more strongly charged than the electrolyte and surface-released counterions (microions).

Furthermore, the colloidal particles and the microions are subject to hydrodynamic interactions (HI). These indirect forces originate from the motion of surrounding macro- and microions, and are transmitted through the intervening fluid. The microion-microion HI forces are referred to in electrolyte theory as the electrophoretic forces [1-5]. The electrophoretic effect on the self-diffusion of a microion is quite small and might be ignored as a first approximation when the microionic diffusion in dilute electrolyte solutions, without immersed colloids, is considered. In contrast to this, colloid-microion and, in particular, colloid-colloid HI do contribute significantly to the self-diffusion of the large colloid particles and can not be ignored. An interesting example highlighting the importance of colloidmicroion HI is given by the tracer diffusion of a single macroion in an electrolyte solution, i.e., by colloidal self-diffusion at practically zero colloid volume fraction $\phi$. In this limiting case, a minimum in $D_{c}^{L}(\phi=0)$, i.e. a maximal electrolyte friction, is observed experimentally when the electrostatic screening length is roughly equal to the diameter of the colloid particle [6-9]. As shown in previous work [10-12], the zero-density value of $D_{c}^{L}$ depends 
crucially on the colloid-microion HI (see also subsection IV A).

The self-diffusion of charged colloidal particles in an electrolyte solution is a long-standing electrokinetic problem with a rich history of research [13-28]. The theoretical approaches used to study this problem usually follow two main routes. The first one is the continuum mechanics approach of standard electrokinetic theory, as exemplified by the classic work of Booth [13] and Ohshima et al. [17]. The electrolyte ions in this approach are treated in a mean-field-like way as charge-densities coupled to the hydrodynamic solvent flow. The hydrodynamic flow field is therefore taken into account explicitly. The resulting electrokinetic equations for the mean densities and the flow field are then typically linearized and solved for boundary conditions specified at infinity and at the boundaries of the colloidal particles [23, 29]. However, in this classic approach mostly a single colloidal particle is considered, although some extensions have been devised to obtain colloidal transport properties in less dilute systems. These extensions, though quite successful in some cases related to electrophoresis and sedimentation [30-32], are often ad-hoc and commonly involve the use of cell models [30, 31, 33] where inter-colloid correlations are ignored.

The second route to obtain colloidal self-diffusion coefficients consists of statistical mechanical theories adapted to colloid dynamics. These theories, in common with the electrokinetic approach, have that the solvent is described as a structure-less hydrodynamic continuum characterized solely by the dielectric constant and the shear viscosity. However, the colloid particles, and possibly the hydrated microions, are treated as individual dynamic entities with their stochastic time evolution described on the Smoluchowski [10, 11, 34-36] or Fokker-Planck (Langevin) [16, 19, 37, 38] levels of description.

In the simplest statistical mechanical theories, the electrolyte friction (EF) influence on colloidal self-diffusion is ignored altogether [39-43]. The suspension is then described as an effective one-component fluid of dressed macroions, interacting at larger distances by a spherically symmetric, screened Coulomb potential. The microions enter into this description only through an effective screening parameter and an effective macroion charge $[22,25,44]$. The one-component macroion fluid model suffices to describe the colloid friction $(\mathrm{CF})$ effect which usually dominates colloidal self-diffusion. A more elaborate variant of the one-component macroion fluid model applied to long-time self-diffusion accounts additionally for the HI between the colloidal particles [45-47]. As a consequence of colloid-colloid HI, rather unexpected features of low-salinity suspensions, or more generally of suspensions 
of particles with long-range repulsive interactions, are observed like the hydrodynamic enhancement of colloidal self-diffusion [47-51], and a non-monotonic density dependence of the so-called hydrodynamic function that describes collective particle diffusion $[52,53]$. In the present work, an additional HI effect will be explained, namely the non-monotonic density dependence of the long-time self-diffusion coefficient observed in low-salt suspensions of colloidal macroions (see section IV).

In more refined multi-component statistical mechanical theories of ion diffusion, the colloidal particles and the electrolyte ions are treated on an equal footing as charged bodies interacting via excluded volume and electrostatic (Coulomb) forces. This refinement allows the study of the combined influence of colloid friction and electrolyte friction on $D_{c}^{L}$. Moreover, non-mean-field effects can be investigated such as finite microion size effects on colloidal self-diffusion [12], and colloid charge reversal as indicated by possible reversal of the electrophoretic mobility of a macroion [54].

Many-component theories describing macroion self-diffusion have been developed, for example by Schurr [15], and by Medina-Noyola and co-workers [19-21, 24]. The latter work is based on a generalized Langevin equation (GLE) description. An extension of the GLE approach to non-dilute suspensions which includes a detailed comparison of CF and EF effects on colloidal self-diffusion, is given in the recent work of Méndez-Alcaraz and Alarcón-Waess [55]. Due to the complexities arising from the inclusion of hydrodynamic interactions, hydrodynamics has generally been unsatisfactorily ignored in these approaches $[15,19-21,24,55]$, or added in an ad-hoc manner by the use of a hydrodynamic rescaling argument originally proposed for colloidal hard spheres [56].

In this paper, we develop a multi-component mode coupling scheme (MCS) for the longtime self-diffusion of colloidal macroions. In this scheme, all ionic species are treated as charged hard spheres undergoing overdamped Brownian motion described by the manyparticle Smoluchowski equation [25, 57]. In particular, rather than ignoring hydrodynamics, we include the HI between all ions on the far-field level. This should be a fair approximation at least for smaller colloid concentrations [25]. Since the microions are much smaller than the colloid spheres, they can be described as point-like with regard to their excluded volume and hydrodynamic interactions (i.e., as Stokeslets) characterized, however, by a finite value of the free diffusion coefficient. As an input for the static pair correlation functions, required by the MCS, we use analytic expressions obtained from the mean spherical approximation 
(MSA) solution specialized to strongly asymmetric electroytes [58-61]. The MSA is the simplest scheme which accounts for the excluded volume of the ions and preserves the exact zeroth and second order moment conditions of local charge neutrality and charge oscillations valid at equilibrium. Using the fact that the microions are far more mobile than the colloid spheres, we derive an expression for $D_{c}^{L}$ particularly suited for practical use.

The present article is a major generalization of previous work by the authors to non-dilute charge-stabilized suspensions. In that previous work the theoretical tools were developed to study long-time transport properties in multi-component systems [62-64], and in which the colloidal electrolyte friction effect was studied in infinitely dilute suspensions with both point-like $[10,11]$ and finite sized electrolyte ions [12]. In [10, 12] it was shown that the effect on colloidal tracer diffusion of the HI between colloids and electrolyte ions (the HI between the electrolyte ions themselves contributes very little) is, firstly, to completely and naturally remove the electrolyte friction contribution due to the excluded-volume interactions between the colloids and electrolyte ions. Secondly, the colloid-microion HI strongly reduces the magnitude of the electrolyte friction. We could also show that the effect of the finite size of the electrolyte ions is only significant, provided the electrolyte ionic strength is not very large, when the ratio of the colloid radius to the electrolyte ion radius is less than about 5.

Our paper is organized as follows: In section II, we give the essentials of the simplified mode coupling scheme of colloidal self-diffusion, and extend the theory to non-zero colloidal concentrations. In section III, we subsequently derive simplified expressions from this scheme for the most common case in which the electrolyte ions are much more mobile than the colloidal particles. Furthermore, we show that the GLE results of macroion diffusion derived in [55], are recovered from our expressions as a special case when HI are ignored and the colloid-colloid correlations are weak. The results of our calculations of $D_{c}^{L}$ as a function of colloid and electrolyte concentrations are discussed in section IV. The dominating effect of the colloidal self-friction is additionally explored using an one-component effective macroion fluid model. To elucidate the physical origin of the unusual non-monotonic density dependence of $D_{c}^{L}$ observed in the mode coupling calculations for low-salinity suspensions, we perform an effective hard-sphere model calculation. Finally, in section $\mathrm{V}$ we present our conclusions. 


\section{MANY-COMPONENT THEORY OF COLLOIDAL SELF-DIFFUSION}

\section{A. Self-diffusion of Brownian particles}

We consider a suspension of charged spherical colloidal particles immersed in a $\mathrm{m}$ component electrolyte solution. The colloidal particles (macroion component $\alpha=0=c$ ) and the small electrolyte ions (microion components $\alpha=1, \ldots, m$ ) are described on an equal footing by a strongly asymmetric primitive model system of uniformly charged hard spheres. The solvent is assumed to be completely structureless and characterized only by its dielectric constant $\epsilon$ and viscosity $\eta_{0}$. The colloidal macroions, in particular, are characterized by their particle radius $a_{c}$, charge $Z e$ (where $e$ is the electron charge), number density $n_{c}$, volume fraction $\phi=(4 \pi / 3) n_{c} a_{c}^{3}$, and free diffusion coefficient $D_{c}^{0}=k_{B} T / \zeta_{c}^{0}$ where $\zeta_{c}^{0}=6 \pi \eta_{0} a_{c}$ is the single-sphere friction coefficient for a fluid sticking to the colloid surface. The counter-ions released from the colloid surfaces form an additional microion component which we assume to be identical to the ions of one of the electrolyte components. The dynamics of all ions is taken to be over-damped so that the configurational probability distribution function is described by the many-body Smoluchowski equation. The associated solvent flow is described by the low-Reynold's number stationary Stokes equation [57].

The long-time self-diffusion coefficient, $D_{c}^{L}=k_{B} T / \zeta_{c}^{L}$, of a colloidal probe sphere can be obtained by calculating the associated long-time friction coefficient $\zeta_{c}^{L}$. This coefficient is larger than the single-particle friction coefficient $\zeta_{c}^{0}$ due to the non-instantaneous response of the neighboring macroions and microions. It is equal to the ratio of the magnitude of an externally applied weak force, $\mathbf{F}$, that acts on the probe sphere only, to the magnitude of the steady-state mean velocity that the particle acquires due to this force. More generally, we recall from linear response theory applied to Smoluchowski dynamics that [10]

$$
\left\langle\mathbf{V}_{c}\right\rangle_{n e q}(t)=\frac{1}{k_{B} T} D_{c}(t) \mathbf{F}=\frac{1}{\zeta_{c}(t)} \mathbf{F}
$$

to first order in $\mathbf{F}$, where $\left\langle\mathbf{V}_{c}\right\rangle_{n e q}(t)$ is the mean velocity of the probe particle that it has acquired during the time interval $t$ after the force has been switched on, and $\langle\ldots\rangle_{n e q}$ denotes a non-equilibrium ensemble average. This equation also serves to define the timedependent self-friction function $\zeta_{c}(t)$ in terms of the self-diffusion function $D_{c}(t)$. For reasons of symmetry $\left\langle\mathbf{V}_{c}\right\rangle_{n e q}(t)$ is collinear with the applied force. The self-diffusion function can be identified with the time derivative, $D_{c}(t)=d W_{c}(t) / d t$, of the colloidal mean-square 
displacement, $W_{c}(t)$, where

$$
W_{c}(t)=\frac{1}{6}\left\langle\left[\mathbf{r}_{c}(t)-\mathbf{r}_{c}(0)\right]^{2}\right\rangle
$$

and $\mathbf{r}_{c}$ is the position vector pointing to the center of the probe sphere. Here $\langle\ldots\rangle$ is the equilibrium average for a system unperturbed by the external force. The mean-square displacement is linear in $t$ at short and long times, as quantified by the short-time and long-time slopes $D_{c}^{S}=D_{c}(t \rightarrow 0)$ and $D_{c}^{L}=D_{c}(t \rightarrow \infty)$, respectively, which obey the order relations $0<D_{c}^{L}<D_{c}^{S} \leq D_{c}^{0}$.

We can relate the self-diffusion function, $D_{c}(t)$, to the so-called self-intermediate scattering function, $G_{c}(q, t)$, defined by $[22,25]$

$$
G_{c}(q, t)=\left\langle\exp \left\{i \mathbf{q} \cdot\left[\mathbf{r}_{c}(t)-\mathbf{r}_{c}(0)\right]\right\}\right\rangle
$$

which is measurable by dynamic light scattering combined with partial refractive index matching of the colloidal spheres. An expansion in the scattering wave number $q$ shows that $G_{c}(q, t)$ is related to the self-diffusion function as

$$
D_{c}(t)=-\lim _{q \rightarrow 0} \frac{1}{q^{2}} \frac{\partial}{\partial t} G_{c}(q, t) .
$$

Using a projection operator technique Nägele et al. $[63,64]$ have derived the exact evolution equation

$$
\frac{\partial}{\partial t} G_{c}(q, t)=-q^{2} D_{c}^{S} G_{c}(q, t)-\int_{0}^{t} d \tau M_{c}^{\mathrm{irr}}(q, t-\tau) \frac{\partial}{\partial \tau} G_{c}(q, \tau)
$$

which relates $G_{c}(q, t)$ to the so-called irreducible self memory function $M_{c}^{\text {irr }}(q, t)$. The integral term in this equation describes the electro-hydrodynamic retardation effect caused by the non-instantaneous response of the surrounding colloids and microions. The initially exponential decay of $G_{c}(q, t)$ is quantified by the short-time self-diffusion coefficient $D_{c}^{S}=k_{B} T / \zeta_{c}^{S}$, which is smaller than $D_{c}^{0}$ when hydrodynamic flow reflections from surrounding particles are significant. On applying Eq. (4) to Eq. (5) we obtain an integral equation for $D_{c}(t)$, that is

$$
D_{c}(t)=D_{c}^{S}-\int_{0}^{t} d \tau M_{c}^{\mathrm{irr}}(0, t-\tau) D_{c}(\tau) .
$$

Since we are interested in the long-time self-diffusion coefficient we specialize this equation to the $t \rightarrow \infty$ limit, leading to

$$
\frac{D_{c}^{L}}{D_{c}^{S}}=\left[1+\frac{\Delta \zeta_{c}^{L}}{\zeta_{c}^{S}}\right]^{-1},
$$


where the relative increase in the colloid particle friction for long-times is expressed as

$$
\frac{\Delta \zeta_{c}^{L}}{\zeta_{c}^{S}}=\int_{0}^{\infty} d t M_{c}^{\mathrm{irr}}(0, t)
$$

where $\zeta_{c}^{L}=\zeta_{c}(t \rightarrow \infty)=\zeta_{c}^{S}+\Delta \zeta_{c}^{L}$.

\section{B. Simplified mode coupling scheme}

The exact microscopic expression for $M_{c}^{\text {irr }}(0, t)$ depends in a complicated way on the electro-steric particle forces, and on the many-body hydrodynamic mobility tensors of all ions. To allow for further progress, some approximations have to be introduced. Nägele et al. [63] have developed a mode-coupling approximation for calculating the long-time behaviour of the self-intermediate scattering function in a multi-component system of particles undergoing over-damped motion. In this theory far-field hydrodynamic interactions are included. We will outline the theory here, paying particular attention to those parts which are relevant for the calculation of the long-time self-diffusion coefficient.

The mode-coupling scheme enters as an approximation for the irreducible memory function. In this approximation, it is assumed that the evolution of the irreducible memory function is dominated mainly by bilinear products of the microscopic number densities of the various components in the system, including the one-particle density of the colloidal probe particle. The number densities are conserved quantities and are therefore, at least for small wave numbers, the slowest variables to relax in the system. We do not detail the derivation of the mode-coupling expression for the irreducible memory function here, which is included in [63], but merely state the resulting expression in the limit $q \rightarrow 0$. This expression is

$$
M_{c}^{\mathrm{irr}}(0, t)=\frac{D_{c}^{S}}{(2 \pi)^{2} n_{c}} \int d \mathbf{k} G_{c}(k, t) \sum_{\gamma, \gamma^{\prime}=0}^{m} V_{c \gamma}(\mathbf{k}) V_{c \gamma^{\prime}}(\mathbf{k}) S_{\gamma \gamma^{\prime}}(k, t)
$$

where the sum goes over all $m+1$ ionic species including the colloidal macroions $(\gamma=0=c)$. The memory function is expressed here in terms of the partial dynamic structure factors, $S_{\gamma \gamma^{\prime}}(k, t)$, which describe dynamic density correlations between ions of components $\gamma$ and $\gamma^{\prime}$. The microscopic expression for the so-called vertex functions, $V_{c \gamma}(\mathbf{k})$, in Eq. (9) depends on the hydrodynamic diffusivity tensors, $\mathbf{D}_{i j}^{\alpha \beta}\left(\mathbf{r}^{N}\right)$, that are complicated functions of the positions, $\mathbf{r}^{N}$, of all $N$ ions. These tensors relate the hydrodynamic force on ion $j \in \beta$ to 
the drift velocity of ion $i \in \alpha$. In this work, we account for the pairwise additive far-field contributions to the diffusivity tensors up to the $O\left(r^{-3}\right)$ level in the inter-particle distance $r$. In this so-called Rotne-Prager approximation, the diffusivity tensors are approximated by $[63,65]$

$$
\mathbf{D}_{i j}^{\alpha \beta}\left(\mathbf{r}^{N}\right) \approx D_{\alpha}^{0} \delta_{i j} \delta_{\alpha \beta} \mathbf{I}+\left(1-\delta_{i j} \delta_{\alpha \beta}\right) \mathbf{T}_{\alpha \beta}^{R P}\left(\mathbf{r}_{i}^{\alpha}-\mathbf{r}_{j}^{\beta}\right),
$$

where $\mathbf{I}$ is the unit tensor. The Rotne-Prager tensor is given by

$$
\mathbf{T}_{\alpha \beta}^{R P}(\mathbf{r})=\frac{k_{B} T}{6 \pi \eta_{0}}\left\{\frac{3}{4 r}[\mathbf{I}+\hat{\mathbf{r}} \hat{\mathbf{r}}]+\frac{a_{\alpha}^{2}+a_{\beta}^{2}}{4 r^{3}}[\mathbf{I}-3 \hat{\mathbf{r}} \hat{\mathbf{r}}]\right\}
$$

where $\hat{\mathbf{r}}=\mathbf{r} / r, a_{\alpha}$ is the ion radius of species $\alpha, D_{\alpha}^{0}$ is the free diffusion coefficient of an $\alpha$-type ion, and $\mathbf{r}_{i}^{\alpha}$ is the position vector pointing to the center of particle $i \in \alpha$. The first term in Eq. (11) is the well-known Oseen tensor for the hydrodynamic interaction between two point particles (Stokeslets). For the large colloidal spheres $D_{c}^{0}$ is given by the StokesEinstein relation $D_{c}^{0}=k_{B} T /\left(6 \pi \eta_{0} a_{c}\right)$. However in the case of point-like electrolyte ions, when $a_{\alpha}$ is set equal to zero, rather than having an infinite free diffusion coefficient, which would remove any effect on the long-time friction from the relaxation of the electrolyte cloud, the ions are taken to have a large but finite free-diffusion coefficient $D_{\alpha}^{0}$. This implies that the (hydrated) microions have an effective radius which nonetheless does not enter into their direct or hydrodynamic interactions.

The mode-coupling theory vertex functions, under the Rotne-Prager approximation for the hydrodynamic interactions, are given by

$$
V_{c \gamma}(\mathbf{k})=-(\hat{\mathbf{q}} \cdot \mathbf{k}) \sum_{\delta=0}^{m} \sqrt{n_{c} n_{\delta}}\left[h_{c \delta}(k)-\frac{1}{D_{c}^{0}} h_{c \delta}^{d}(k)\right] S_{\gamma \delta}^{-1}(k),
$$

where $n_{\delta}$ is the number density of species $\delta$, and $h_{c \delta}(k)$ is the spatial Fourier transform of the partial total correlation function, $h_{c \delta}(r)$, between a colloidal ion and an ion of component $\delta$. The function $S_{\gamma \delta}^{-1}(k)$ is the $\gamma \delta$ component of the inverse of the matrix of partial static structure factors, $S_{\alpha \beta}(k)=\delta_{\alpha \beta}+\sqrt{n_{\alpha} n_{\beta}} h_{\alpha \beta}(k)$. Finally, the function $h_{c \delta}^{d}(k)$ is the distinct part of the partial hydrodynamic function, $H_{c \delta}(k)$, between a colloid particle and a $\delta$-component ion. The partial hydrodynamic functions are defined as

$$
H_{\alpha \beta}(k)=\delta_{\alpha \beta} D_{\alpha}^{S}+\sqrt{n_{\alpha} n_{\beta}} h_{\alpha \beta}^{d}(k),
$$

where $D_{\alpha}^{S}=\left\langle\hat{\mathbf{k}} \cdot \mathbf{D}_{11}^{\alpha \alpha} \cdot \hat{\mathbf{k}}\right\rangle$ is the short-time self-diffusion coefficient of an $\alpha$-type ion. The distinct part of $H_{\alpha \beta}(k)$ is

$$
h_{\alpha \beta}^{d}(k)=V\left\langle\hat{\mathbf{k}} \cdot \mathbf{D}_{12}^{\alpha \beta}\left(\mathbf{r}^{N}\right) \cdot \hat{\mathbf{k}}\right\rangle,
$$


with $V$ denoting the system volume. The partial hydrodynamic functions quantify the configuration-averaged effect of the hydrodynamic interactions on the short-time suspension dynamics. In the Rotne-Prager approximation $\mathbf{D}_{i i}^{\alpha \alpha}=D_{\alpha}^{0} \mathbf{I}$, which implies that the shorttime self-diffusion coefficients are simply set equal to the free-diffusion coefficients. This is a valid approximation for charge-stabilized systems at sufficiently small colloid and electrolyte concentrations [25]. Moreover, under the Rotne-Prager approximation the partial distinct hydrodynamic functions can be written as [12]

$$
h_{\alpha \beta}^{d}(k)=\int_{a_{\alpha}+a_{\beta}}^{\infty} d r r h_{\alpha \beta}(r) K_{\alpha \beta}(k, r)-\frac{2 k_{B} T}{3 \eta_{0}}\left(a_{\alpha}^{2}+a_{\beta}^{2}+3 a_{\alpha} a_{\beta}\right) \frac{j_{1}\left(k\left[a_{\alpha}+a_{\beta}\right]\right)}{k\left[a_{\alpha}+a_{\beta}\right]},
$$

where the integrand kernel is given by

$$
K_{\alpha \beta}(k, r)=\frac{k_{B} T}{\eta_{0}}\left\{j_{0}(k r)-\frac{j_{1}(k r)}{k r}+k^{2}\left(a_{\alpha}^{2}+a_{\beta}^{2}\right) \frac{j_{2}(k r)}{3(k r)^{2}}\right\} .
$$

The functions $j_{n}(x)$ are $n$-th order spherical Bessel functions of the first kind. In a previous article [12] we have derived a technique to calculate the integral for $h_{\alpha \beta}^{d}(k)$ based on a class of integrals of the Laplace transforms $\tilde{G}_{\alpha \beta}(s)=\mathcal{L}\left\{r g_{\alpha \beta}(r)\right\}$, where $g_{\alpha \beta}(r)=1+h_{\alpha \beta}(r)$ is the radial distribution function between $\alpha$ and $\beta$ ions. This technique changes the kernel of the integrals from the set of oscillatory functions, $j_{n}(x)$, to low-order polynomials. The integrals tend to be better behaved and furthermore reduce the level of integrations we must perform by one, since we do not need to compute the radial distribution functions first. Analytic expressions of $\widetilde{G}_{\alpha \beta}(s)$ have been provided by Blum and Høye within the mean spherical approximation for a general multi-component primitive model system [59]. These expressions are used in our calculations of $D_{c}^{L}$.

In describing the HI on the far-field level, we neglect all hydrodynamic flow reflections while preserving the positive definiteness of the matrix of the exact diffusivity tensors. The most significant hydrodynamic contribution to the magnitude of $D_{c}^{L}$ comes from the colloidcolloid HI. However, due to the strong electrical repulsion, close configurations of colloidal macroions are unlikely, so that the Rotne-Prager approximation of $\mathbf{D}_{i j}^{c c}$ is applicable, provided $\phi$ and the electrolyte concentration are not so large that significant many-body HI contributions come into play (i.e., $\phi \lesssim 0.1$ ). Furthermore, it has been shown previously [12] that the effect of the finite size of the electrolyte ions on $D_{c}^{L}$ is significant only when the colloid/electrolyte ion size ratio is typically smaller than 5 . We expect this to be the case also in non-dilute suspensions. We therefore ignore the size of the electrolyte ions and treat 
them as point-like particles characterized by finite free diffusion coefficients. In the limiting case of a single tracer macroion being pulled through a solution of point-like microions, the $O\left(r^{-3}\right)$ far-field expression for the colloid-microion diffusivity tensor becomes exact, even when a point ion touches the surface of the tracer sphere. Thus, although the Rotne-Prager form of the hydrodynamic interactions between the colloids and the point-like ions is not exact in the case of a non-dilute suspension, we expect the contribution to $D_{c}^{L}$ from the neglected near-field HI to be quite small. Inclusion of near-field HI contributions and lubrication forces arising from small but finite-sized microions would complicate our mode coupling scheme tremendously. Furthermore, as far as the microion-microion and colloidmicroion HI are concerned, it is questionable whether the lubrication theory of continuum mechanics is applicable at length scales comparable to the size of a water molecule, or to the magnitude of the colloidal surface roughness, for example when a hydrated counterion is close to a colloid surface.

Having specified the approximations used for the partial hydrodynamic functions, we are in the position to substitute the Rotne-Prager approximation vertex functions from Eq. (12) into Eq. (9). Introducing the $(m+1)$ component column vectors $\mathbf{h}_{c}^{d}(k)$ and $\mathbf{h}_{c}(k)$ having components $\sqrt{n_{\alpha}} h_{c \alpha}^{d}(k)$ and $\sqrt{n_{\alpha}} h_{c \alpha}(k)$, respectively, we obtain a mode coupling scheme (MCS) expression for the long-time additional colloid friction, which includes the effects of HI on the far-field level. This expression is

$$
\begin{aligned}
\frac{\Delta \zeta_{c}^{L}}{\zeta_{c}^{0}}=\frac{D_{c}^{0}}{6 \pi^{2}} \int_{0}^{\infty} d k k^{4}\left[\mathbf{h}_{c}(k)-\frac{1}{D_{c}^{0}} \mathbf{h}_{c}^{d}(k)\right] \cdot \mathbf{S}^{-1}(k) \times & \\
& \left\{\int_{0}^{\infty} d t G_{c}(k, t) \mathbf{S}(k, t)\right\} \cdot \mathbf{S}^{-1}(k) \cdot\left[\mathbf{h}_{c}(k)-\frac{1}{D_{c}^{0}} \mathbf{h}_{c}^{d}(k)\right],
\end{aligned}
$$

where we have used that $\zeta_{0}^{S}=\zeta_{c}^{0}$ in the Rotne-Prager approximation. This equation depends on a number of unknown quantities: the static correlation functions, which act as input into the theory, and the dynamic correlation functions, $G_{c}(k, t)$ and $\mathbf{S}(k, t)$. Here, $\mathbf{S}(k, t)$ is the matrix of partial dynamic structure factors, $S_{\alpha \beta}(k, t)$, and $\mathbf{S}(k)=\mathbf{S}(k, 0)$.

A similar, though far more complicated mode-coupling expression has been derived by Nägele et al. for the $S_{\alpha \beta}(q, t)$ of Brownian mixtures [64]. This expression is an adaption to overdamped Brownian systems of the standard mode-coupling expression for the collective memory function of simple liquids [66] and atomic mixtures [67, 68], derived originally by Götze and collaborators. Far-field HI are included in the scheme derived in [64] on assuming 
that the dissipative hydrodynamic interactions are coupled to the particle densities, and by using the same factorization of the four-point dynamic density correlator for hydrodynamically interacting particles as is employed in the mode-coupling theory of atomic particles. The MCS in [64] includes thus the standard mode-coupling theory of non-hydrodynamically interacting colloidal spheres (which do not exist in reality) as a limiting case [69, 70]. In the MCS of Nägele et al. far-field HI have a non-trivial effect on the form of the vertex functions, with the consequence that the location of a possible glass transition point can be affected. In this respect the scheme differs substantially from other methods to incorporate HI into the mode-coupling theory of one-component suspensions, that essentially amount to rescaling the memory equation for $S(q, t)$ without $\mathrm{HI}$ by the short-time collective [71] or the short-time self-diffusion coefficients [72], the latter coefficients calculated with HI included. To our opinion there is no reason to assume that HI have no influence at all on the location of a non-equilibrium glass transition point, at least for non hard-sphere-like systems that are not dominated by excluded volume interactions. To use short-time hydrodynamic rescaling as an approximate tool for the incorporation of $\mathrm{HI}$ into the calculation of long-time transport properties was proposed originally by Medina-Noyola [56]. In fact it works quite well for dense suspensions of hard spheres, where near-field HI prevail and where particles are strongly caged by neighboring ones $[43,71]$. However, as we will argue in subsection II B, it is not applicable to charge-stabilized suspensions of strongly repelling particles whose excluded volume interactions are masked by long-range electrostatic repulsions.

In combining the MCS equations derived in [64] for the $S_{\alpha \beta}(q, t)$ with the MCS equation for the self-intermediate scattering functions, $D_{c}^{L}$ could be computed self-consistently. This however, would be a very major undertaking, particularly for a many-component Brownian system with far-field hydrodynamic interactions included. For a charge-stabilized suspension of colloidal particles that is not strongly correlated we expect that it is reasonably accurate to solve the coupled equations only in a single iterative step, in which case the short-time forms of $G_{c}(k, t)$ and $\mathbf{S}(k, t)$ can serve as the initial input solution. This expectation is supported by fully self-consistent mode-coupling calculations without HI, that have been performed for the time-integrated dynamic structure factor using the one-component macroion fluid model applied to systems with added salt [42]. In these systems of moderately interacting particles, the overall decay of the dynamic structure factor was found to be only mildly underestimated when the simplified scheme is used $[42,46]$. 
The simplified solution scheme with the short-time correlation functions as input will not be applicable for strongly interacting macroions such as in a high volume fraction suspension at low salt concentrations. Therefore, and for the reason of analytical tractability, we will limit ourselves to suspensions of weakly to moderately charged macroions in which the colloidal volume fraction will not be greater than 0.1 .

The short-time forms of $G_{c}(k, t)$ and $\mathbf{S}(k, t)$, valid within the far-field treatment of $\mathrm{HI}$, are given by

$$
G_{c}(k, t)=e^{-k^{2} D_{c}^{0} t}
$$

and

$$
\mathbf{S}(k, t)=e^{-k^{2} \mathbf{H}(k) \cdot \mathbf{S}^{-1}(k) t} \cdot \mathbf{S}(k),
$$

where $\mathbf{H}(k)$ is the matrix of partial hydrodynamic functions $H_{\alpha \beta}(k)$ with self-part $D_{\alpha}^{S}=D_{\alpha}^{0}$, and where the distinct part is determined by Eq. (15). The short-time form of $\mathbf{S}(k, t)$ is a sum of $m$ exponentially decaying diffusive modes, augmented by a so-called Debye mode characterized by a relaxation rate which is non-zero in the $k \rightarrow 0$ limit $[10,73]$.

Substituting these short-time expressions into Eq. (17) and performing the time integral, we obtain the remarkably symmetric expression for the incremental long-time friction coefficient

$$
\begin{aligned}
\frac{\Delta \zeta_{c}^{L}}{\zeta_{c}^{0}}=\frac{1}{6 \pi^{2}} \int_{0}^{\infty} d k & k^{2} \times \\
& {\left[\mathbf{h}_{c}(k)-\frac{1}{D_{c}^{0}} \mathbf{h}_{c}^{d}(k)\right] \cdot\left[\mathbf{S}(k)+\frac{1}{D_{c}^{0}} \mathbf{H}(k)\right]^{-1} \cdot\left[\mathbf{h}_{c}(k)-\frac{1}{D_{c}^{0}} \mathbf{h}_{c}^{d}(k)\right] . }
\end{aligned}
$$

This simplified MCS expression is one major result of the present paper. It requires the partial correlation functions $h_{\alpha \beta}(k)$ and $h_{\alpha \beta}^{d}(k)$ as input. For this purpose, we use the analytical MSA expression for the Laplace transform, $\widetilde{G}_{\alpha \beta}(s)$, of $r g_{\alpha \beta}(r)$ of a mixture consisting of moderately charged colloidal particles and point-like microions [59]. The input $h_{\alpha \beta}(k)$ is then determined through

$$
h_{\alpha \beta}(k)=-\frac{4 \pi}{k} \operatorname{Im}\left\{\widetilde{G}_{\alpha \beta}(s=i k)\right\}
$$

and the distinct hydrodynamic functions $h_{\alpha \beta}^{d}(k)$ are conveniently expressed in terms of a simple integral over $\widetilde{G}_{\alpha \beta}(s)$ using a calculation technique explained in [12]. The MSA solution of the primitive model is known to produce non-physical negative contact values for dilute but strongly interacting like-charged ions due to its poor description of short-range 
correlations. Nonetheless, we believe that the MSA is a useful, consistent and above all analytical theory of the static structure in a multi-component system of charged bodies that can be used without concern in situations when the macroion surface charge density is reasonably small. The MSA was also used as static input in a recent study of ionic selfdiffusion in simple electrolytes, where a mode coupling approach, with HI disregarded, was used in combination with dynamic density functional theory [74].

While Eq. (20) includes the incremental long-time friction contributions to $D_{c}^{L}$ from all ionic interactions, including those between the point-like ions themselves, we can reduce the computational effort required to calculate $D_{c}^{L}$ considerably, on exploiting the fact that the microions are usually far more mobile than the large colloid particles.

\section{COLLOID AND ELECTROLYTE FRICTION}

The contribution to the long-time friction increment of a colloidal macroion due to the other colloidal macroions alone, i.e., the colloid friction $(\mathrm{CF})$, can be found by ignoring all the terms in equation (20) in which microion-microion and colloid-microion correlation functions are present. This is equivalent to retaining only the zeroth-order terms in the smallness parameters, $D_{i}^{0} / D_{c}^{0}$, with $i=1, \ldots, m$. In this limiting case of infinitely mobile microions, the electrolyte atmosphere around each macroion remains spherically symmetric at each instance of time, so that the electrolyte friction contribution to $\Delta \zeta_{c}^{L}$ vanishes.

Using the simplifying notation $S(k)=1+n_{c} h(k)$ and $H(k)=D_{c}^{0}+n_{c} h^{d}(k)$ for the colloid-colloid partial static structure factor and partial hydrodynamic function, we obtain the colloid-friction contribution to $\Delta \zeta_{c}^{L}$,

$$
\left[\frac{\Delta \zeta_{c}^{L}}{\zeta_{c}^{0}}\right]^{C F}=\frac{1}{6 \pi^{2} n_{c}} \int_{0}^{\infty} d k k^{2} \frac{\left[S(k)-H(k) / D_{c}^{0}\right]^{2}}{S(k)+H(k) / D_{c}^{0}} .
$$

This is the same expression one obtains when the simplified mode-coupling scheme, with far-field HI included, is applied to a one-component model of colloidal spheres [46]. In earlier work the expression in Eq. (22) has been used, in conjunction with the effective one-component macroion fluid model, to estimate the long-time self-diffusion coefficient of charged colloidal spheres. It's application has led to the prediction of hydrodynamic enhancement of self-diffusion in low-salinity systems [46, 47], an effect that has been studied since then in various experimental and computer simulation work [48-51]. 
Regarding the virial expansion of $D_{c}^{L}$ for a suspension of neutral hard spheres, with

$$
D_{c}^{L}=1-\alpha_{H S} \phi+O\left(\phi^{2}\right)
$$

a value $\alpha_{H S}=3 / 16$ for the first virial coefficient is predicted using Eq. (22). This value differs from the exact numerical result 2.1, obtained from a full account of near-field HI. Contrary to charge-stabilized spheres, near-field HI is dominant in suspensions of neutral spheres where contact configurations are likely. The error made in $\alpha_{H S}$ is partially due to neglecting near-field HI in Eq. (22), and partially to the mode coupling approximation itself which does not reproduce the exact low-density memory function of neutral spheres. Without HI, the exact value for $\alpha_{H S}$ is 2 , whereas the value $4 / 3$ is obtained from Eq. (22) when specialized to zero HI where $h^{d}(k)=0$ and $H(k)=D_{c}^{0}$.

Having identified the colloidal friction contribution, it is clear that the remaining longtime friction, due to the electrolyte ion kinetics, is given by the difference between the total (incremental) friction experienced by a colloidal probe sphere, and the colloid friction contribution. That is

$$
\left[\frac{\Delta \zeta_{c}^{L}}{\zeta_{c}^{0}}\right]^{E F}=\frac{\Delta \zeta_{c}^{L}}{\zeta_{c}^{0}}-\left[\frac{\Delta \zeta_{c}^{L}}{\zeta_{c}^{0}}\right]^{C F}
$$

In the following, we analyze the electrolyte friction contribution to $D_{c}^{L}$ for very mobile microions.

\section{A. Expressions for very mobile microions}

For the vast majority of charge-stabilized colloidal systems, the colloidal particles are at least an order of magnitude larger in size than the hydrated electrolyte ions. This size disparity implies that the microion free-diffusion coefficient, $D^{0}$, is at least one order of magnitude greater than the colloidal free diffusion coefficient.

Therefore, we may expand the mode coupling result in Eq. (20) in powers of the smallness parameter $\delta$, defined by

$$
\delta=\frac{D_{c}^{0}}{D_{c}^{0}+D^{0}},
$$

and to retain friction contributions only up to linear order in $\delta$. For simplicity, we assume that all electrolyte ion free diffusion coefficients are equal to $D_{0}$. Our choice of the smallness parameter is suggested by the form of the central matrix inverse appearing in the integrand of Eq. (20). Note here that $\delta=D_{c}^{0} / D^{0}$ to first order in $D_{c}^{0} / D^{0}$. 
On writing the expansion

$$
\frac{\Delta \zeta_{c}^{L}}{\zeta_{c}^{0}}=\left(\frac{\Delta \zeta_{c}^{L}}{\zeta_{c}^{0}}\right)_{0}+\delta\left(\frac{\Delta \zeta_{c}^{L}}{\zeta_{c}^{0}}\right)_{1}+O\left(\delta^{2}\right)
$$

we note first that the zeroth-order term is given by the colloid friction term explicated in Eq. (22). To obtain the first order term in $\delta$, which according to Eq. (24) is identical to the leading-order electrolyte friction contribution, it is convenient to partition all matrices appearing in the integrand of Eq. (20) into macroion-macroion, macroion-microion and microion-microion block components. Collecting all terms linear in $\delta$ leads then, after some algebra, to

$$
\left[\frac{\Delta \zeta_{c}^{L}}{\zeta_{c}^{0}}\right]^{E F}=\delta \frac{2}{3 \pi^{2}} \sum_{i=1}^{m} n_{i} \int_{0}^{\infty} d k k^{2}\left\{\frac{h_{c i}(k) H(k)-h_{c i}^{d}(k) S(k)}{D_{c}^{0} S(k)+H(k)}\right\}^{2}+O\left(\delta^{2}\right),
$$

where the sum extends over all electrolyte ion species. It should be noted here that the integrand is actually independent of $D_{c}^{0}$, since $H(k)$ and $h_{T i}^{d}(k)$ are proportional to $D_{c}^{0}$. This expression for $\left(\Delta \zeta_{c}^{L}\right)^{E F}$, together with Eq. (22) for $\left(\Delta \zeta_{c}^{L}\right)^{C F}$, is computationally much easier to use than the full expression given by Eq. (20), since there is no need to perform the cumbersome matrix inversion. Moreover, it is interesting to note that in the firstorder expression for the electrolyte friction the microion-microion HI does not contribute, only contributing to quadratic order in $\delta$. In contrast to this, the microion-microion static correlations do contribute indirectly to the linear order term since they are coupled to the colloid correlation functions via the Ornstein-Zernike equations. Due to their simplicity, Eqs. (27) and (22) are used in most of the subsequent calculations.

\section{B. Excluded volume contribution to electrolyte friction}

To quantify the influence of HI on the self-diffusion of charged colloidal spheres, we compare with calculations of $D_{c}^{L}$ where HI are fully ignored. Without HI all distinct partial hydrodynamic functions, $h_{c \alpha}^{d}(k)$, in Eqs. (22) and (27) are equal to zero, giving

$$
\left(\frac{\Delta \zeta_{c}^{L}}{\zeta_{c}^{0}}\right)_{\text {no HI }}=\frac{n_{c}}{6 \pi^{2}} \int_{0}^{\infty} d k k^{2} \frac{h^{2}(k)}{1+S(k)}+\delta \sum_{i=1}^{m} \frac{2 n_{i}}{3 \pi^{2}} \int_{0}^{\infty} d k k^{2}\left[\frac{h_{c i}(k)}{1+S(k)}\right]^{2}+O\left(\delta^{2}\right) .
$$

This zero-HI mode coupling result should be compared to the earlier work of Méndez-Alcaraz and Alarcón-Waess [55] where long-time self-diffusion in non-dilute charge-stabilized suspensions has been studied using a multi-component generalized Langevin equation approach developed by Medina-Noyola and co-workers [20, 24, 56]. The GLE approach of Medina-Noyola 
is similar in spirit to our MCS, but HI is disregarded from the outset. In the simplified variant of the GLE approach used by Méndez-Alcaraz and Alarcón-Waess, the long-time friction increment is approximated by

$$
\left(\frac{\Delta \zeta_{c}^{L}}{\zeta_{c}^{0}}\right)^{G L E}=\frac{1}{6 \pi^{2}} \sum_{\alpha=0}^{m} \frac{D_{c}^{0}}{D_{c}^{0}+D_{\alpha}^{0}} n_{\alpha} \int_{0}^{\infty} d k k^{2} h_{c \alpha}^{2}(k) .
$$

This expression follows from Eq. (28) (using $D_{\alpha}^{0}=D^{0}$ for $\alpha>0$ ) when the colloid-colloid structure factor is put equal to one in the denominator. Although it has been derived by a GLE method different from our mode coupling approach, Eq. (29) can be considered as a low-density approximation of Eq. (28) for the case of very weakly correlated colloidal macroions.

As we will show subsequently, neglecting HI leads to a significant overestimation of the electrolyte friction contribution. Ignoring $\mathrm{HI}$ in particular, results in the occurrence of a spurious excluded volume contribution to the electrolyte friction part. This excluded volume contribution is due to the short-range hard-core interactions between the colloidal spheres and the point-like microions, and remains present even if the system is uncharged $[10,21]$. It would be a genuine physical effect if there would be no HI. To obtain the excluded volume friction we evaluate Eq. (20) using Eq. (24) for zero HI and for uncharged macroand microparticles. An expansion in the colloid volume fraction leads then to

$$
\left(\frac{\Delta \zeta_{c}^{L}}{\zeta_{c}^{0}}\right)_{\text {neutral }}^{E F}=\delta \frac{1}{3} \Omega_{c} n+O(\phi)
$$

where $\Omega_{c}=(4 \pi / 3) a_{c}^{3}$ is the volume of a colloidal sphere, and $n=n_{1}+\ldots+n_{m}$ is the total number density of point-like microions. In comparison to this, we recall that $\left(\Delta \zeta_{c}^{L}\right)^{C F}=$ $(4 / 3) \phi \zeta_{c}^{0}+O\left(\phi^{2}\right)$ for the colloid friction part of an uncharged system without HI. At large electrolyte concentration the first term on the right-hand-side of Eq. (30) is so large that a non-physical monotonic descent of $D_{c}^{L}(\phi=0)$ is predicted as a function of the electrolyte concentration. This is in conflict with experimental observation where a minimum in $D_{c}^{L}(\phi=$ 0 ) is observed when the electrolyte double layer extension is roughly equal to the colloid diameter [7]. However when far-field HI is accounted for in Eq. (20) we instead obtain after some algebra that

$$
\left(\frac{\Delta \zeta_{c}^{L}}{\zeta_{c}^{0}}\right)_{\text {neutral }}^{E F}=0+O\left(\phi^{2}\right) .
$$

Hence, with HI there is in fact no excluded volume contribution to electrolyte friction up to quadratic order in $\phi$. In physical terms the disappearance of the zeroth-order excluded 
volume friction part is due to the fore-aft symmetry of the Stokes flow field created by a single moving sphere. This flow advects the point-like microions with the stream-lines of the sphere. In earlier GLE work on single-macroion elecrolyte friction, it has been rightly argued by Vizcarra-Rendon et al. [21] that the excluded volume contribution caused by the neglect of $\mathrm{HI}$ should be absorbed into the bare colloid friction coefficient $\zeta_{c}^{0}$, since the microions are distinguished from the solvent molecules only in terms of their long-range electrostatic interactions. This amounts to a calculation of $D_{c}^{L}$ without HI, with $\Delta \zeta_{c}^{L}$ in

Eq. (7) substituted by $\Delta \zeta_{c}^{L}-\left(\Delta \zeta_{c}^{L}\right)_{\text {neutral }}^{E F}$. This subtraction of the excluded volume term has in fact been tacitly used in the work of Méndez-Alcaraz and Alarcón-Waess [55]. However as we will show, it is not sufficient to correct satisfactorily for the overestimation of the electrolyte friction and colloid friction, which has its origin in the neglect of HI.

\section{Single macroion diffusion}

The MCS expression in Eq. (20), with the static input in MSA, simplifies considerably when the self-diffusion of an isolated macroion is considered. In this limiting case, there is no colloid friction contribution to $D_{c}^{L}(\phi=0)$, and the MSA solution simplifies to the Debye-Hückel form of the static correlation functions. The full electrolyte friction is then given by

$$
\begin{aligned}
\left(\frac{\Delta \zeta_{c}^{L}}{\zeta_{c}^{0}}\right)_{n_{c}=0}^{E F}=\delta & \frac{2 l_{B} Z^{2}}{3 \pi}\left(\frac{K}{1+K}\right)^{2} \times \\
& \int_{0}^{\infty} d y \frac{y^{2} w_{c}(y ; K)^{2}}{\left[y^{2}+K^{2}\right]\left[y^{2}+(1-\delta) K^{2}+(3 / 2) \delta K\left(y^{2}+K^{2}\right) f_{O}(y / K)\right]}
\end{aligned}
$$

with

$$
w_{c}(y ; K)=\frac{3}{2}\left[y^{2}+K^{2}\right] f_{R P}(y ; K)-\left[\cos (y)+K j_{0}(y)\right]
$$

Here, $Z$ is the macroion charge number, $K=\kappa a_{c}$ and $l_{B}=L_{B} / a_{c}$, with $L_{B}=e^{2} /\left(\epsilon k_{B} T\right)$, are the Debye-Hückel screening parameter, and the Bjerrum length of the solvent, respectively, expressed in units of the colloid radius. The far-field macroion-electrolyte HI effect is included through the function

$$
f_{R P}(y ; K)=\int_{1}^{\infty} d x e^{-K(x-1)}\left[j_{0}(x y)-\frac{j_{1}(x y)}{x y}+\frac{j_{2}(x y)}{3 x^{2}}\right]
$$


whereas the HI between the point-like microions are described by the Oseen function

$$
f_{O}(t)=\frac{1}{t^{3}}\left[t+\left(t^{2}-1\right) \arctan (t)\right]
$$

The quadratic dependence on $Z$ derives from the Debye-Hückel input. The Oseen function in Eq. (32) is multiplied by the smallness paramater $\delta$. Therefore, to first order in $\delta$, microion-microion HI can be neglected as noted already in the discussion of Eq. (27).

A small- $K$ expansion of Eq. (32) gives Onsager's limiting law,

$$
\left(\frac{\Delta \zeta_{c}^{L}}{\zeta_{c}^{0}}\right)_{n_{c}=0}^{E F}=\frac{1}{3} l_{B} Z^{2} K(1-d)+O\left(K^{2}\right),
$$

valid for a dilute electrolyte solution that forms an extended double layer around the central macroion. Here, $d^{2}=1-\delta$ implying that $1-d=\delta / 2+O\left(\delta^{2}\right)$. Thus, our simplified mode coupling scheme has the desirable feature to include Onsager's expression as a limiting case. HI enter the friction coefficient first in the quadratic order term in $K$, as also observed in the case of simple electrolytes $[75,76]$.

The large- $K$ behavior of $\left(\Delta \zeta_{c}^{L}\right)^{E F}$ by contrast, is strongly influenced by HI. From an asymptotic analysis of Eq. (32), we obtain

$$
\left(\frac{\Delta \zeta_{c}^{L}}{\zeta_{c}^{0}}\right)_{n_{c}=0}^{E F}=\delta \frac{l_{B} Z^{2}}{K^{4}}+O\left(\delta^{2}\right) .
$$

This finding should be compared to the full zero-HI result,

$$
\left(\frac{\Delta \zeta_{c}^{L}}{\zeta_{c}^{0}}\right)_{n_{c}=0}^{E F}=\delta \frac{l_{B} Z^{2}}{6} \frac{K}{(1+K)^{2}}+\delta \frac{1}{3} \Omega_{c} n+O\left(\delta^{2}\right),
$$

which follows from Eq. (32) with $f_{R P}$ and $f_{O}$ set equal to zero. Thus Onsager's expression is recovered from the zero-HI expression, but a much slower $1 / K$ decay is predicted for large $K$. This qualitative difference in the large- $K$ asymptotics with and without HI has profound consequences when suspensions of macroions with fixed electrostatic surface potential are considered. We will discuss this in the following section.

\section{RESULTS}

The numerical results included in this section have been obtained using a three-component primitive model consisting of colloidal spheres of charge-number $Z$, immersed in a 1-1 electrolyte of point-like microions with salt concentration $n_{s}$. The monovalent counter-ions 
dissociated from the colloid surfaces are assumed to be identical to the salt anions, giving a total counter-ion concentration equal to $n_{s}+n_{c}|Z|$. The solvent is taken to be water at $T=293 \mathrm{~K}$, corresponding to a Bjerrum length of $L_{B} \approx 0.71 \mathrm{~nm}$. We account for the self-dissociation of water by placing a lower limit of $n_{s}=10^{-6} \mathrm{M}$ on the excess salt concentration. To be specific, we choose $a_{c}=6.9 \mathrm{~nm}$ which is a representative macroion radius of a suspension of moderately charged globular proteins like Apoferritin [77]. Using the StokesEinstein relation for stick fluid boundary conditions, this gives $D_{c}^{0}=3.11 \times 10^{-11} \mathrm{~m}^{2} / \mathrm{s}$. Unless stated differently, the ratio of free-diffusion coefficients between the microions and the colloidal spheres is taken to be $D^{0} / D_{c}^{0}=51.6$, which corresponds to the free diffusion coefficient $D^{0}=1.60 \times 10^{-9} \mathrm{~m}^{2} / \mathrm{s}$ as an average of the measured free-diffusion coefficients of the single atom ions $\mathrm{Na}^{+}$and $\mathrm{Cl}^{-}$in water [4]. All system parameters have been selected such that the colloidal particles are only moderately correlated, with the peak height of the colloid structure factor $S(q)$ being smaller than 1.5. This justifies our use of the simplified MCS.

The three partial radial distribution functions are generated using the analytic MSA expression given in [59], which consist of a hard-sphere like part, an electrostatic part and a cross term according to

$$
\widetilde{G}_{\alpha \beta}(s)=\widetilde{G}_{\alpha \beta}^{H S}(s)+\widetilde{G}_{\alpha \beta}^{E L}(s)+\Delta \widetilde{G}_{\alpha \beta}(s) .
$$

The Laplace transform solution depends on the MSA screening parameter $\Gamma$ which, in units of $a_{c}$, is determined as the unique positive solution of the fourth-order algebraic equation $[59,60]$

$$
4 \Gamma^{2}=K^{2}+\frac{3 \phi l_{B} Z^{2}}{(1+C+2 \Gamma)^{2}},
$$

where $C=3 \phi /(1-\phi)$ and $K=\kappa a_{c}$. Here, $\kappa$ is the Debye-Hückel microion screening parameter,

$$
\kappa^{2}=4 \pi L_{B}\left(2 n_{s}+n_{c}|Z|\right)=\kappa_{s}^{2}+\kappa_{c i}^{s},
$$

which consists of a salt-ion part, and a part due to the surface-released counter-ions. At small colloid volume fractions, where $C \ll 1$, one is allowed to neglect the cross term contribution to $\widetilde{G}_{\alpha \beta}(s)$ [59]. In the Debye-Hückel limit $n_{c} \rightarrow 0, \Gamma$ reduces to $K / 2$. For neutral colloidal spheres in an electrolyte solution, where $Z=0$, the MSA solutions for $g_{c c}(r)$ and $g_{c i}(r)$ reduce to the Percus-Yevick radial distribution functions for a mixture of hard spheres and neutral point particles. 


\section{A. Single-colloid electrolyte friction at constant surface potential}

We discuss first the most simple case of a single macroion $\left(n_{c}=0\right)$ to show that it can be non-physical to discuss electro-kinetic friction without including the effects of HI, as was done for example in $[15,20,55]$. To give an example where the neglect of HI fails most dramatically, consider a tracer macroion with fixed electrostatic surface potential, $\psi_{0}$, rather than fixed charge. Such a system has been purportedly explored experimentally in [8]. In the Debye-Hückel approximation, the relationship between surface potential and charge is given by

$$
\beta e \psi_{0}=l_{B} \frac{Z}{1+K},
$$

so that a constant potential requires the colloid charge to increase linearly with increasing screening parameter $K$. According to Eq. (37), which describes electrolyte friction without HI, this would lead to the non-physical result that

$$
\left[\frac{\Delta \zeta_{c}^{L}}{\Delta \zeta_{c}^{0}}\right]^{E L}-\left[\frac{\Delta \zeta_{c}^{L}}{\Delta \zeta_{c}^{0}}\right]_{\text {neutral }}^{E L} \sim K .
$$

That is, the friction coefficient would diverge linearly for an increasingly thin double layer, even after the spurious excluded volume has been removed. The excluded volume term itself diverges like $K^{1 / 2}$ for large $K$, due to its linear dependence on the salt concentration. The large- $K$ divergence is in contradiction to experimental findings and to physical expectation. It is however confirmed by the MCS calculations with HI included, according to which the electrolyte friction coefficient (diffusion coefficient $D_{c}^{L}$ ) always goes through a maximum (minimum) with increasing $K$, regardless of whether the colloid particle is kept at constant charge or constant potential. As a matter of fact when HI is considered according to Eq. (32),

the incremental friction coefficient decays asymptotically like $K^{-2}$ for fixed potential and like $K^{-4}$ for fixed charge, in accord with the physical expectation that the Stokes-Einstein free diffusion coefficient must be recovered at high salinity.

Our discussion is exemplified in Fig. 1 showing MCS results with and without HI both for fixed charge and potential. The zero-HI curves are the result with the excluded volume term removed from the friction. While a zero-HI calculation for fixed macroion charge still leads to a maximum in the electrolyte friction at $K=1$ (cf. Eq. (37)), its height and location are strongly overestimated. With HI the friction maximum is located instead at $K \approx 0.3$. It should be mentioned here that the friction coefficient predicted by our MCS is 
nearly coincident to the corresponding result obtained from the Booth-Geigenmüller theory of single-macroion electrolyte friction $[12,13,18]$.

\section{B. Primitive model calculation with colloid and electrolyte friction}

In the following we present numerical MCS results for the colloidal long-time self-diffusion coefficient at non-zero colloid volume fractions. The long-time incremental friction experienced by a probe macroion is calculated via expressions (22) and (27), for the CF and EF contributions respectively. For all systems considered we have checked that the results are practically indistinguishable from the ones obtained from the full mode-coupling expression given by Eq. (20), even down to a mobility ratio, $D^{0} / D_{c}^{0}$, of 10 . In Fig. $2(\mathrm{a}), D_{c}^{L}$ is plotted against the added salt concentration for a set of volume fractions. It is clear from the figure that $D_{c}^{L}$ is primarily affected by the interactions between the colloids. The additional EF causes only a minor decrease in $D_{c}^{L}$ that is especially small at larger $\phi$. The monotonic increase of $D_{c}^{L}$ when salt is added (for $\phi>10^{-5}$ ) is due to the growing screening of the electrostatic repulsion between the macroions caused by the salt ions. At larger $\phi$, more salt needs to be added to reach the regime where screening is no longer dominated by the surface-released counter-ions, and to observe a strong increase in $D_{c}^{L}$. At high salt content $D_{c}^{L}$ becomes approximately equal to the Stokes-Einstein free-diffusion coefficient, except for the highest studied volume fraction of $\phi=0.1$, where the observed reduction in $D_{c}^{L}$ is due to $\left(\Delta \zeta_{c}^{L}\right)^{C F}\left(\kappa a_{c} \gg 1\right)$ being approximately equal to $3 / 16 \phi$ due to hard-sphere repulsion. By comparison, the high-salt contribution of the EF is negligibly small since $\left(\Delta \zeta_{c}^{L}\right)^{E F}\left(\kappa a_{c} \gg 1\right)=O\left(\phi^{2}\right)$. We recall here however, that the Rotne-Prager approximation of colloid-colloid HI becomes less reliable at large salinity since then near-field HI come into play. Fig. 2(b) shows the corresponding results for $D_{c}^{L}$ when the colloid-microion mobility ratio is enlarged by a factor of five. It is only for such a weak mobility asymmetry that EF also becomes significant for larger values of $\phi$.

Another interesting feature of long-time self-diffusion is observed in the low salt regime. On imagining a vertical cut in the plots of (a) and (b) at a low salt concentration, it can be seen that $D_{c}^{L}$ decreases first with $\phi$ for low $\phi$ but after reaching a local minimum, it increases when $\phi$ is further increased. The non-monotonic $\phi$-dependence of $D_{c}^{L}$ at low salinity appears counterintuitive, although it is a genuine physical effect. On first sight one would expect a 
monotonic decay of $D_{c}^{L}$ with increasing $\phi$ as observed experimentally in many cases. The surprising non-monotonicity of $D_{c}^{L}$ will be explored further in subsection IV C, via a onecomponent macroion model, since it is only due to the interactions between the colloids. The electrolyte friction becomes smaller with increasing $\phi$ not only relative to the colloid friction, since the latter vanishes at zero volume fraction, but also in absolute terms. In Fig. 3, we show the electrolyte friction corresponding to Fig. 2(a). Quite interestingly, the electrolyte friction decreases at essentially all salt concentrations with increasing $\phi$, despite the increasing concentration of surface-released counter-ions. This behavior of the EF can be explained physically as the effect of a levelling of the electrolyte background, which occurs because with more colloids per volume, there must be increased sharing of the available electrolyte ions. The effect, then, of the relaxation of inhomogeneities in the electrolyte background is necessarily reduced in magnitude. Therefore, the electrolyte friction is largest at $\phi=0$ which is still a small effect even at its maximum. The height of the maximum decreases with increasing colloid-microion size ratio. As a result, electrolyte friction will generally be insignificant in (non-dilute) suspensions of charge-stabilized colloidal particles that are either reasonably large or not too strongly charged. In other words, highly charged and mobile colloids or biomolecules suspended at sufficiently low salinity and volume fraction, are required to observe a significant electrolyte friction effect. The uprise in $\left(\Delta \zeta_{c}^{L}\right)^{E F}$ at high salinity, as noticed for the most concentrated system with $\phi=0.1$, is due to the excluded volume contribution $\left(\Delta \zeta_{c}^{L}\right)^{E F}\left(\kappa a_{c} \gg 1\right)$ which is of $O\left(\phi^{2}\right)$.

To explore the importance of HI, we now discuss MCS predictions for $D_{c}^{L}$ where the influence of HI has been ignored. Fig. 4(a) shows the result for $D_{c}^{L}$ both with and without the EF included. In the former case, the zero-charge excluded volume part, $\left(\Delta \zeta_{c}^{L}\right)_{\text {neutral }}^{E F}$, has been removed. As can be seen, the EF effect without HI is much stronger than when HI is included, in particular around the salt concentration where the minimum in $D_{c}^{L}(\phi=0)$ occurs. Moreover, the comparison with the results in Fig. 2 shows that the colloid-colloid far-field HI has the effect to enlarge $D_{c}^{L}$ significantly, in particular for low salt concentrations. We further notice from this comparison that the non-monotonic volume fraction dependence of $D_{c}^{L}$ gets lost when the HI are disregarded. This shows that the non-monotonicity in $D_{c}^{L}$ is due to colloid-colloid HI, a finding that we will explore in more detail in the following subsection. Fig. 4(b) displays zero-HI results for $D_{c}^{L}$ obtained after inclusion of the excluded volume electrolyte friction. As can be seen for larger salinity, this additional friction 
contribution strongly overcompensates the decrease in the colloid friction originating from electrostatic screening, leading to a non-physical precipitous decline in $D_{c}^{L}$ with increasing salt concentration.

We have found that the electrolyte friction is substantially overestimated when HI are ignored. The same conclusion is valid with regard to colloid friction. In Fig. 5, we plot the CF coefficient calculated with and without HI. In the former case, the Oseen and RotnePrager levels of approximation have been used, respectively. The far-field HI of a charged probe sphere with the surrounding macroions tend to advect the neighboring colloids so that the Brownian motion of the probe sphere is eased. Therefore, the CF is significantly lowered by the far-field HI. Near-field HI, by contrast, leads to an enhanced friction for hard-sphere like systems where it becomes dominant (see e.g. [51]). The colloid friction calculated at the Oseen and Rotne-Prager levels of approximations, respectively, is nearly the same up to $\phi=0.05$, illustrating the dominant influence of the far-field HI. Clearly different values for the colloid friction are found, however, at $\phi=0.1$. This indicates that $\phi=0.1$ is really an upper bound beyond which near-field contributions should be additionally taken into account. The same conclusion applies incidentally to accelerated Stokesian dynamics simulation results for the short-time diffusion in charge-stabilized colloidal suspensions [52]. We further notice from Fig. 5, that the dipolar HI part of the Rotne-Prager tensor has the effect to somewhat decrease the hydrodynamic enhancement of self-diffusion caused by the leading order Oseen part.

\section{One-component model calculation}

We have shown that the influence of the electrolyte kinetics on the colloidal self-diffusion is generally quite small for charge-stabilized systems where the ratio $D^{0} / D_{c}^{0}$ is reasonably large. In such cases, where the CF dominates we can use a much simpler effective onecomponent model of dressed macroions to study the long-time diffusion of colloids. It should be stressed however that the electrolyte ions still have a large overall effect on the colloid dynamics through the screening of the effective interactions between the colloids and the consequent static inter-particle structure. In what follows we employ the one-component model to study in more detail the non-monotonic density dependence of $D_{c}^{L}$ observed for low salinity. 
In the effective one-component model the colloidal particles are described as uniformly charged spheres interacting via an effective pair-potential [23, 44]

$$
\frac{u(r)}{k_{B} T}=L_{B} Z^{2}\left(\frac{e^{\kappa a_{c}}}{1+\kappa a_{c}}\right)^{2} \frac{e^{-\kappa r}}{r}, \quad r>2 a_{c}
$$

with $\kappa$ given by Eq. (40). The charge number $Z$ appearing in the present one-component model is an effective one, and must be distinguished from the bare macroion charge appearing in the multi-component primitive model that we have used so far. For non-weakly charged colloids, the effective macroion charge is smaller than the bare one due to non-linear screening effects. We are not concerned here with the ongoing discussion on how the two charges are related to each other. This is a challenging many-body problem that has been addressed by a variety of methods $[61,78,79]$. Only approximate answers have been given to date and they depend significantly on the invoked approximations.

Since our concern here is to understand the physical origin of the non-monotonicity in $D_{c}^{L}$, we merely use Eq. (43) for a given $Z$ to derive the static input, $S(k)$, that is needed as the input to the MCS colloid friction expression given in Eq. (22). Within the one-component model we can use an improved static input without having to worry about Stillinger-Lovett moment conditions imposed on the partial static correlation functions of ionic mixtures. Therefore, we use the rescaled mean spherical approximation (RMSA) solution of $h(k)$ [80] assuming $Z$ to be independent of $\phi$. By using the RMSA in place of the MSA we can be assured that $g(r)$ remains non-negative at all $r$. Furthermore, to extend the range of applicability of Eq. (22) to higher concentrations where near-field HI matters and the inequality $D_{c}^{S}<D_{c}^{0}$ is found, we replace the $D_{c}^{0}$ appearing in Eq. (22) by $D_{c}^{S}$, and calculate the colloid hydrodynamic function and $D_{c}^{S}$ using the (zeroth-order) $\delta \gamma$ method due to Beenakker and Mazur [81]. This method accounts approximately for many-body near-field HI through socalled ring-diagrams. Through comparison with recent Stokesian Dynamics simulations of charge-stabilized spheres, it has been shown to give quite reliable predictions of $H(q)$ also for high concentrations $[52,53,77]$.

The results of our one-component MCS calculations with HI included are shown in Fig. 6. At the lowest considered amount of added 1-1 electrolyte, the non-monotonicity of $D_{c}^{L}$ as a function of $\phi$ is most pronounced, with a local minimum appearing at $\phi \approx 5 \times 10^{-3}$, and a maximum at $\phi \approx 0.1$. The steep decline in $D_{c}^{L}$ at high volume fractions is due to steric inter-particle repulsion which becomes stronger with increasing $\phi$. The minimum and 
the subsequent maximum, on the other hand, is due to the interplay between the leadingorder Oseen HI contribution which enhances self-diffusion, and the long-range electro-static repulsion at low screening. At larger $n_{s}$ the particles can approach closer to each other so that the dipolar part of the far-field HI (decaying like $r^{-3}$ ) and eventually also near-field HI come into play, and which both counteract the diffusion enhancement. Therefore, the minimum in $D_{c}^{L}$ will disappear at larger salinity as observed in the figure. Moreover, our calculations show that the non-monotonic behavior of $D_{c}^{L}$ gets weaker, for fixed charge, when the particle radius is increased. For comparison, Fig. 6 also includes results for $D_{c}^{L}$ where HI has been ignored. As one expects $D_{c}^{L}$ decays monotonically without HI for all $\phi$ considered even for very low amounts of added salt.

In [55] it has been suggested that the influence of $\mathrm{HI}$ on $D_{c}^{L}$ may be accounted for in an ad-hoc way through the hydrodynamic rescaling prescription, $\left(D_{c}^{L}\right)_{\mathrm{HI}} \approx\left(D_{c}^{L}\right)_{\mathrm{noHI}}\left(D_{c}^{S}\right)_{\mathrm{HI}}$, proposed by Medina-Noyola originally for suspensions of hard spheres [56]. This rescaling procedure and its straightforward extensions to other long-time transport properties, work quite well in the case of concentrated suspensions of neutral hard spheres, where the manybody near-field HI dominate [43]. However, it fails in the case of low-salinity charge-stabilized suspensions where, due to $D_{c}^{S} \leq D_{c}^{0}$, it predicts a reduction in the magnitude of $D_{c}^{L}$ instead of the diffusion enhancement observed in experiment, theory and simulation.

\section{Spherical-annulus model}

To check that the non-monotonic $\phi$-dependence in $D_{c}^{L}$ is not an artefact of the simplified MCS, and to elucidate this hydrodynamic effect further, we now employ an effective hard sphere (EHS) model, also referred to as the spherical annulus model [82, 83]. This model consists of particles that interact directly via hard-sphere interactions specified by an effective diameter, $\sigma^{\text {EHS }}$, that is larger than the hydrodynamic diameter, $\sigma=2 a_{c}$, which in turn determines the hydrodynamic interactions. For a sufficiently large size ratio $X=\sigma^{\text {EHS }} / \sigma \gtrsim 3$, the HI can be treated to very good accuracy on the pairwise additive far-field level, since near-field HI is precluded by the distance of closest approach. Furthermore, as an additional simplification applicable to dilute systems only, the colloid radial distribution function is 
assumed to be simply a step function,

$$
g^{E H S}(r)= \begin{cases}0, & \text { for } r<\sigma^{E H S} \\ 1, & \text { for } r \geq \sigma^{E H S}\end{cases}
$$

The spherical annulus model serves as a highly simplified, qualitative model for dilute suspensions of particles which interact through long-range repulsive forces, such as in charged colloids at low salt concentrations, where the strong double layer repulsion ensures that the probability of two particles being close to each other is very low. Using a regular perturbation expansion in the smallness parameter $\sigma / \sigma^{\mathrm{EHS}}=1 / X$, Cichocki and Felderhof have shown that the long-time self-diffusion coefficient in this model is given by [82]

$$
\frac{D_{c}^{L}}{D_{c}^{0}}=1-\left[2 X^{3}-\frac{33}{16} X^{2}+\frac{567}{2560} X+O\left(X^{0}\right)\right] \phi,
$$

where $\phi=(\pi / 6) n_{c} \sigma^{3}$, and where it has been assumed that $\phi X^{3} \ll 1$. The first term in the brackets is due to the excluded-volume interactions describing the electrostatic repulsion. The next two terms arise from the Oseen approximation of HI with irrelevant smaller contributions of $O\left(X^{0}\right)$ left out.

Considering equation (45) qualitatively, we see that the first term due to the excludedvolume interactions tends to decrease $D_{c}^{L}$ as the range of the direct interactions increases, while the negative-valued second term originating from the Oseen contribution, tends to enhance the self-diffusion. This enhancement is slightly softened by the weaker HI contribution coming from the third term proportional to $X$.

For a quantitative comparison with the MCS results in Fig. 6, a method is required to determine the effective hard sphere diameter as a function of $\phi$. To this end we use a standard method for charge-stabilized systems [23] where $\sigma^{E H S}$ is defined as the distance at which the effective potential energy is equal to the thermal energy, i.e., $\beta u\left(\sigma^{E H S}\right)=1$. This amounts to solving the transcendental equation

$$
\kappa \sigma^{E H S} e^{\kappa \sigma^{E H S}}=\kappa L_{B} Z^{2}\left(\frac{e^{\kappa a_{c}}}{1+\kappa a_{c}}\right)^{2}
$$

for $\sigma^{E H S}$, which is easily achieved numerically. The inverse of the function on the lefthand side is known as Lambert's W or Product-Log function. For strongly charged spheres $\exp \{-\beta u(r)\}$ increases steeply from zero to one around $r \approx \sigma^{E H S}$.

The EHS results for $D_{c}^{L}$ calculated via the Product-Log definition of $\sigma^{E H S}$ are shown in Fig. 7. A minimum in $D_{c}^{L}$ is observed at $\phi \sim 10^{-2}$ for the lowest salt content considered. As 
for the MCS results, the minimum becomes increasingly shallow with growing salt concentration, and when the particle size is increased for fixed $Z$. Furthermore, neglecting HI leads again to a monotonic $\phi$-dependence of $D_{c}^{L}$. Thus the spherical annulus model supplies an independent confirmation of hydrodynamic enhancement, and of the non-monotonic density dependence of $D_{c}^{L}$, as predicted by the MCS for colloidal particles which repel each other over long distances. The substantial difference in the values of $D_{c}^{L}$ calculated via the MCS and the schematic spherical annulus model, can be attributed to the very crude zero-density step-function approximation of $g(r)$, which ignores all higher-order inter-particle correlations, and to an ambiguity in defining $\sigma^{E H S}$. The spherical annulus model is not applicable at larger volume fractions $(\phi \sim 0.1)$ or higher salt content $\left(n_{s} \sim 10^{-2} \mathrm{M}\right)$, since the underlaying assumption that $X(\phi)$ is appreciable larger than one is not fulfilled in these cases (see the results for $X(\phi)$ plotted in Fig. 7).

Finally, we point out that a non-monotonic behavior of the long-time self-diffusion coefficient, explainable by the hydrodynamic effect discussed above, was indeed found in recent fluorescence correlation spectroscopy measurements by Wilk et al. [28] on short DNA fragments. A comparison with these experimental data, however, is outside the general scope of this paper and will be made elsewhere [84].

\section{CONCLUSIONS}

Using a recently developed multi-component mode-coupling theory for Brownian suspensions, we have developed a versatile scheme to calculate the colloidal long-time self-diffusion coefficient of interacting charge-stabilized particles. This scheme describes the colloid and electrolyte friction effects on an equal footing. We have used this scheme to quantify the kinetic friction effect of the electrolyte ions, where for the first time HI has been included in non-dilute systems, and we have explored the parameter range where it is most significant. We have shown that the electrolyte friction contribution to $D_{c}^{L}$ is usually insignificant relative to the colloid friction, provided that the mobility difference between the colloidal spheres and the electrolyte ions is large and the suspension is not very dilute. The inclusion of HI is important for a valid description both of the electrolyte and colloid friction effect. Ignoring HI, as was done in the earlier GLE approach [55], results in a substantial overestimation of the electrolyte friction and, for smaller values of $\phi$, also of the colloid friction. In particular, 
for dilute systems with a constant electrostatic surface potential a non-physical behavior of $D_{c}^{L}(\phi=0)$ is observed when the colloid-microion HI are disregarded. Additionally we find that the leading-order far-field HI between charged colloidal particles which repel each other over larger distances, causes an enhancement of self-diffusion that can give rise to a nonmonotonic volume fraction dependence of $D_{c}^{L}$. The non-monotonic density dependence of $D_{c}^{L}$ was studied in more detail using the effective one-component macroion fluid model and the more schematic spherical annulus model, and it is found in all the investigated model systems when HI is included.

The present simplified MCS is applicable to suspensions of moderately correlated particles only. The general trends however, can also be expected to remain valid for a fluid-like suspension of strongly correlated particles. In future work, we plan to use an improved static input obtained from more elaborate hybrid integral equation schemes [85, 86] where the small-distance static correlations between macroions and (finite-sized) microions are described more accurately. This will allow us to study the influence of non-linear correlation effects on $D_{c}^{L}$ such as the effect of colloidal charge-reversal. As a final remark, we note that the present MCS can be extended to determine additional colloidal transport properties characterizing charge-stabilized suspensions such as the collective diffusion coefficient, where electrokinetic effects can be expected to be larger than for self-diffusion [77], and the colloidal electrophoretic mobility in non-dilute suspensions. Work on these additional transport properties is in progress.

\section{Acknowledgments}

This work has been supported by the Deutsche Forschungsgemeinschaft (Project section B2 of SFB-TR6).

[1] L. Onsager and S. K. Kim, J. Phys. Chem. 61, 215 (1957).

[2] R. M. Fuoss and L. Onsager, J. Phys. Chem. 67, 621 (1963).

[3] H. L. Friedman, Physica 30, 537 (1964).

[4] R. Mills and V. M. M. Lobo, Self-Diffusion in Electrolyte Solutions, vol. 36 of Physical Sciences Data (Elsevier, Amsterdam, 1989). 
[5] J. M. G. Barthel, H. Krienke, and W. Kunz, Physical Chemistry of Electrolyte Solutions, vol. 5 of Topics in Physical Chemistry (Springer, Darmstadt, 1998).

[6] S. Gorti, L. Plank, and B. W. Ware, J. Chem. Phys. 81, 909 (1984).

[7] G. A. Schumacher and T. G. M. van de Ven, Faraday Discuss. Chem. Soc. 83, 75 (1987).

[8] D. N. Petsev and N. D. Denkov, J. Colloid Interface Sci. 149, 329 (1992).

[9] M. Tirado-Miranda, C. Haro-Perez, M. Quesada-Perez, J. Callejas-Fernandez, and R. HidalgoAlvarez, J. Colloid Interface Sci. 263, 74 (2003).

[10] M. Kollmann and G. Nägele, J. Chem. Phys. 113, 7672 (2000).

[11] M. Kollmann and G. Nägele, Europhys. Lett. 52, 474 (2000).

[12] M. G. McPhie and G. Nägele, J. Phys.: Condens. Matter 16, S4021 (2004).

[13] F. Booth, J. Chem. Phys. 22, 1956 (1954).

[14] B. J. Berne and R. Pecora, Dynamic Light Scattering (John Wiley, New York, 1976).

[15] J. M. Schurr, Chem. Phys. 45, 119 (1980).

[16] W. Hess and R. Klein, Adv. Phys 32, 173 (1983).

[17] H. Ohshima, T. W. Healy, R. W. O'Brien, and L. R. White, J. Chem. Soc. Faraday Trans. 2 80, 1299 (1984).

[18] U. Geigenmueller, Chem. Phys. Letters 110, 666 (1984).

[19] M. Medina-Noyola and A. Vizcarra-Rendón, Phys. Rev. A 32, 3596 (1985).

[20] H. Ruiz-Estrada, A. Vizcarra-Rendón, M. Medina-Noyola, and R. Klein, Phys. Rev. A 34, $3446(1986)$.

[21] A. Vizcarra-Rendón, H. Ruiz-Estrada, M. Medina-Noyola, and R. Klein, J. Chem. Phys. 86, $2976(1987)$.

[22] P. N. Pusey, Liquids, Freezing and the Glass Transition: II (North-Holland, Amsterdam, 1989), pp. 763-942.

[23] W. B. Russel, D. A. Saville, and W. R. Schowalter, Colloidal Dispersions (Cambridge University Press, Cambridge, 1991).

[24] G. C. de León, M. Medina-Noyola, O. Alarcón-Waess, and H. Ruiz-Estrada, Chem. Phys. Lett. 207, 294 (1993).

[25] G. Nägele, Phys. Rep. 272, 215 (1996).

[26] J. Horbach and D. Frenkel, Phys. Rev. E 64, 061507 (2001).

[27] A. Evilevitch, V. Lobaskin, U. Olsson, P. Linse, and P. Schurtenberger, Langmuir 17, 1043 
(2001).

[28] A. Wilk, J. Gapinski, A. Patkowski, and R. Pecora, J. Chem. Phys. 121, 10794 (2004).

[29] R. J. Hunter, Zeta Potential in Colloid Science (Academic Press, London, 1981).

[30] H. Ohshima, J. Colloid Interface Sci. 188, 481 (1997).

[31] H. Ohshima, J. Colloid Interface Sci. 195, 137 (1997).

[32] J. Ennis, A. A. Shugai, and S. L. Carnie, J. Colloid Interface Sci. 223, 37 (2000).

[33] F. Carrique, F. J. Arroyo, and A. V. Delgado, J. Colloid Interface Sci. 227, 212 (2000).

[34] E. C. Zhong and H. L. Friedman, J. Phys. Chem. 92, 1685 (1988).

[35] M. Jardat and P. Turq, Z. Phys. Chem. 218, 699 (2004).

[36] J.-F. Dufrêche, O. Bernard, S. Durand-Vidal, and P. Turq, J. Phys. Chem. B 109, 9873 (2005).

[37] T. J. Murphy, J. Chem. Phys. 56, 3487 (1972).

[38] T. J. Thacher, J. I. Lin, and C. Y. Mou, J. Chem. Phys. 81, 2053 (1984).

[39] J. A. Leegwater and G. Szamel, Phys. Rev. A 46, 1999 (1992).

[40] H. Löwen and G. Szamel, J. Phys.: Condens. Matter 5, 2295 (1993).

[41] J. Bergenholtz and N. J. Wagner, Physica A 235, 34 (1997).

[42] A. J. Banchio, G. Nägele, and J. Bergenholtz, J. Chem. Phys. 113, 3381 (2000).

[43] A. J. Banchio, J. Bergenholtz, and G. Nägele, Phys. Rev. Lett. 82, 1792 (1999).

[44] E. J. M. Verwey and J. T. G. Overbeek, Theory of the Stability of Lyophobic Colloids (Elsevier, Amsterdam, 1948).

[45] M. Venkatesan, C. S. Hirtzel, and R. Rajagopalan, J. Chem. Phys. 82, 5685 (1985).

[46] G. Nägele and P. Baur, Physica A 245, 297 (1997).

[47] G. Nägele and P. Baur, Europhys. Lett. 38, 557 (1997).

[48] K. Zahn, J. M. Méndez-Alcaraz, and G. Maret, Phys. Rev. Lett. 79, 175 (1997).

[49] R. Pesché and G. Nägele, Phys. Rev. E 62, 5432 (2000).

[50] W. Härtl, J. Wagner, C. Beck, F. Grierschner, and R. Hempelmann, J. Phys.. Condens. Matter 12, A287 (2000).

[51] G. Nägele, M. Kollmann, R. Pesché, and A. J. Banchio, Mol. Phys. 100, 2921 (2001).

[52] A. J. Banchio, J. Gapinski, A. Patkowski, W. Häußler, A. Fluerasu, S. Saccana, P. Holmqvist, G. Meier, M. P. Lettinga, and G. Nägele, Phys. Rev. Lett. 96, 138303 (2006).

[53] J. Gapinski, A. Patkowski, A. J. Banchio, P. Holmqvist, G. Meier, M. P. Lettinga, and G. Nägele, J. Chem. Phys. 126, 104905 (2007). 
[54] M. Quesada-Pérez, E. Gonzáles-Tovar, A. Martín-Molina, M. Lozada-Cassou, and R. HidalgoÁlvarez, Colloids and Surfaces A 267, 24 (2005).

[55] J. M. Mèndez-Alcaraz and O. Alarcón-Waess, Physica A 268, 75 (1999).

[56] M. Medina-Noyola, Phys. Rev. Lett. 60, 2705 (1988).

[57] J. K. G. Dhont, An Introduction to the Dynamics of Colloids (Elsevier, Amsterdam, 1996).

[58] M. Gillan, B. Larsen, M. P. Tosi, and N. H. March, J. Phys. C: Solide State Phys. 9, 889 (1976).

[59] L. Blum and J. S. Høye, J. Phys. Chem. 81, 1311 (1977).

[60] K. Hiroike, Mol. Phys. 33, 1195 (1977).

[61] L. Belloni, J. Chem. Phys. 85, 519 (1986).

[62] G. Nägele and J. Bergenholtz, J. Chem. Phys. 108, 9893 (1998).

[63] G. Nägele and J. K. G. Dhont, J. Chem. Phys. 108, 9566 (1998).

[64] G. Nägele, J. Bergenholtz, and J. K. G. Dhont, J. Chem. Phys. 110, 7037 (1999).

[65] J. Rotne and S. Prager, J. Chem. Phys. 50, 4831 (1969).

[66] W. Götze and L. Sjögren, Rep. Prog. Phys. 55, 241 (1992).

[67] J. L. Barrat and A. Latz, J. Phys.: Condens. Matter 2, 4289 (1990).

[68] J. S. Thakur and J. Bosse, Phys. Rev. A 43, 4378 (1991).

[69] G. Szamel and H. Löwen, Phys. Rev. A 44, 8215 (1991).

[70] E. Flenner and G. Szamel, Phys. Rev. E 72, 031508 (2005).

[71] M. Fuchs and M. R. Mayr, Phys. Rev. E 60, 5742 (1999).

[72] E. G. D. Cohen, R. Verberg, and I. M. de Schepper, Physica A 251, 251 (1998).

[73] A. Z. Akcasu, B. Hammouda, T. P. Lodge, and C. C. Han, Macromolecules 17, 759 (1984).

[74] J.-F. Dufrêche, O. Bernard, P. Turq, A. Mukherjee, and B. Bagchi, Phys. Rev. Lett. 88, $095902(2002)$.

[75] C. Y. Mou, T. S. Thacher, and J. Lin, J. Chem. Phys. 79, 957 (1983).

[76] E. C. Zhong and H. L. Friedman, J. Sol. Chem. 16, 337 (1987).

[77] J. Gapinski, A. Wilk, A. Patkowski, W. Häussler, A. J. Banchio, R. Pecora, and G. Nägele, J. Chem. Phys. 123, 054708 (2005).

[78] S. Alexander, P. M. Chaikin, P. Grant, G. J. Morales, and P. Pincus, J. Chem. Phys. 80, 5776 (1984).

[79] L. Bocquet, E. Trizac, and M. Aubouy, J. Chem. Phys. 117, 8138 (2002). 
[80] J.-P. Hansen and J. B. Hayter, Mol. Phys. 46, 747 (1982).

[81] C. W. J. Beenakker and P. Mazur, Physica 126, 349 (1984).

[82] B. Cichocki and B. U. Felderhof, J. Chem. Phys. 94, 556 (1991).

[83] A. J. Khair and J. F. Brady, J. Fluid Mech. 557, 73 (2006).

[84] M. G. McPhie, A. Wilk, J. Gapinski, A. Patkowski, R. Pecora, and G. Nägele (2007), work in progress.

[85] M. D. Carbajal-Tinoco and P. González-Mozuelos, J. Chem. Phys. 117, 2344 (2002).

[86] G. I. Guerrero-García, E. González-Tovar, M. Lozada-Cassou, and F. de J. Guerara-Rodríguez, J. Chem. Phys. 123, 034703 (2005). 
FIG. 1: Long-time electrolyte friction experienced by an isolated colloidal macroion of radius $a_{c}=20 \mathrm{~nm}$ under conditions of constant charge $Z=165$ (thin lines) or constant potential (thick lines), both with HI (solid) and without HI (dashed). The electrolyte friction without $\mathrm{HI}$ diverges linearly in $K=\kappa a_{c}$ for constant potential, visible as an exponential in the linear-log plot. In the fixed potential case, $\psi_{0}$ is specified by Eq. (41) with $K=0$ and $Z=165$. The diffusivity ratio is $\delta=0.0078$.

FIG. 2: (a) Long-time macroion self-diffusion coefficient at fixed charge $Z=10, a_{c}=6.9$ $\mathrm{nm}$, and $D^{0} / D_{c}^{0}=51.6$, versus added salt concentration, for colloid volume fractions as indicated. Solid lines: results with $\mathrm{CF}$ and with EF included; dashed lines: without electrolyte friction. (b) Same as in (a), but for $D^{0} / D_{c}^{0}=10.3$.

FIG. 3: Salt-dependence of the electrolyte friction contribution $(\Delta \zeta)^{E L}$, for $Z=10$ and $D^{0} / D_{c}^{0}=51.6$. The upper horizontal scale displays the part of the screening parameter due to added salt.

FIG. 4: Colloidal long-time self-diffusion coefficient calculated while ignoring HI, for the same parameters as in Fig. 2. (a) Solid lines: result for $D_{c}^{L}$ with both $\mathrm{CF}$ and EF included, but without the excluded volume EF contribution. Dashed lines: result including CF only. (b) The solid lines are the same as in (a). The dashed-dotted lines are the result for $D_{c}^{L}$ where, in addition to $\mathrm{CF}$ and $\mathrm{EF}$, the excluded volume EF term is also included.

FIG. 5: Effect of HI on $\Delta \zeta^{C F}$ for $\phi=0.01$ (thin lines) and $\phi=0.05$ (thick lines). The macroion charge number is $Z=10$. The solid, dashed and dashed-dotted lines are results, respectively, using Rotne-Prager HI, Oseen HI, and no HI.

FIG. 6: Long-time self-diffusion coefficient versus $\phi$, calculated using the one-component macroion fluid model with effective charge number $Z=10, a_{c}=6.9 \mathrm{~nm}$, and added salt concentrations as indicated. The RMSA is used for the static input, and $H(q)$ is determined using the $\delta \gamma$ scheme. Solid lines: results with HI; dashed lines: without HI.

FIG. 7: Long-time self-diffusion coefficient corresponding to Fig. 6, obtained from the spher- 
ical annulus model with (solid lines) and without (dashed lines) far-field HI, using the Product-Log definition of $\sigma^{E H S}$. Two different salt concentrations are considered. The right vertical axis displays the scale for $\sigma^{E H S} / \sigma$ (dotted lines). The effective diameter decreases monotonically with increasing $\phi$. 
FIG. 1:

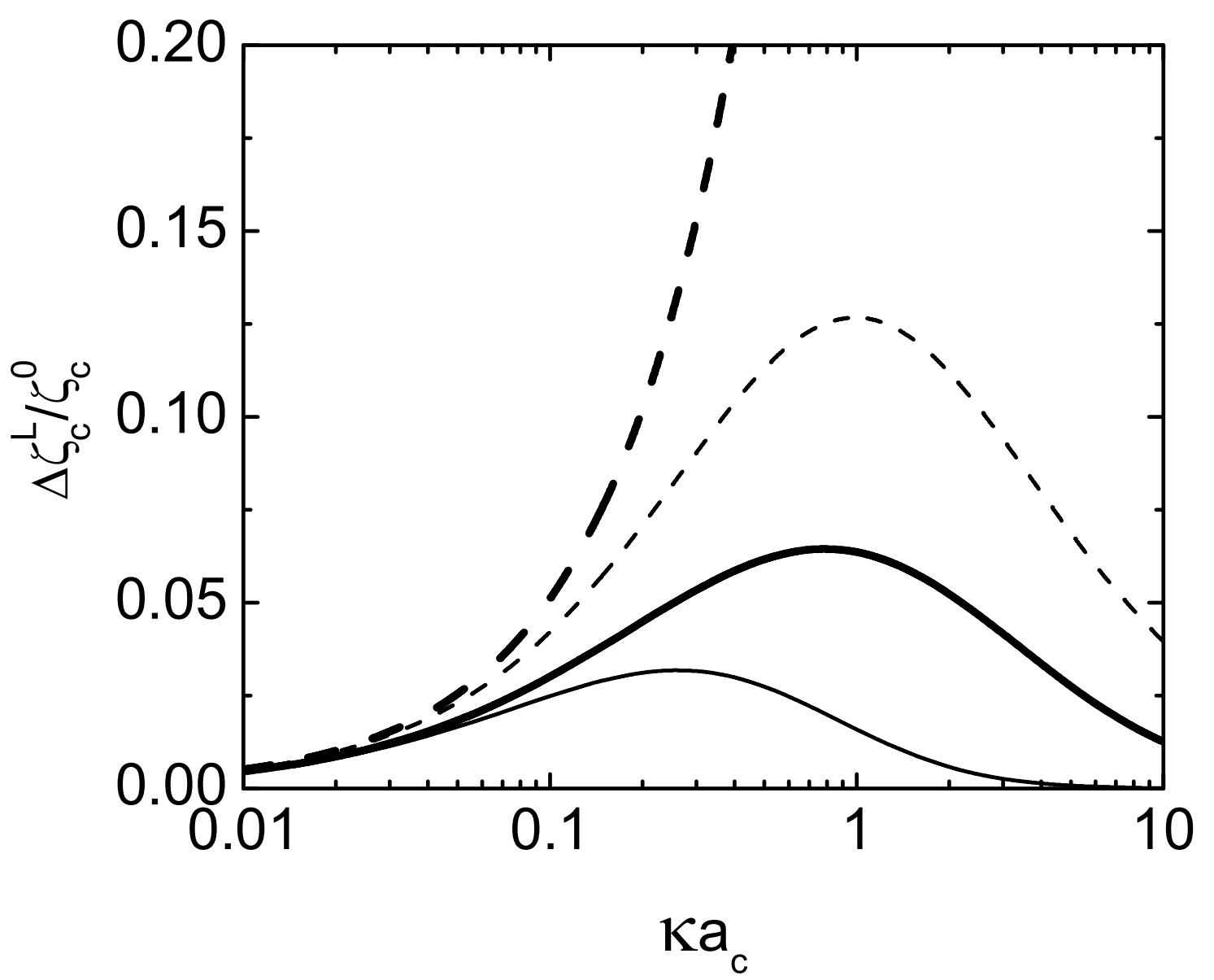


FIG. 2:

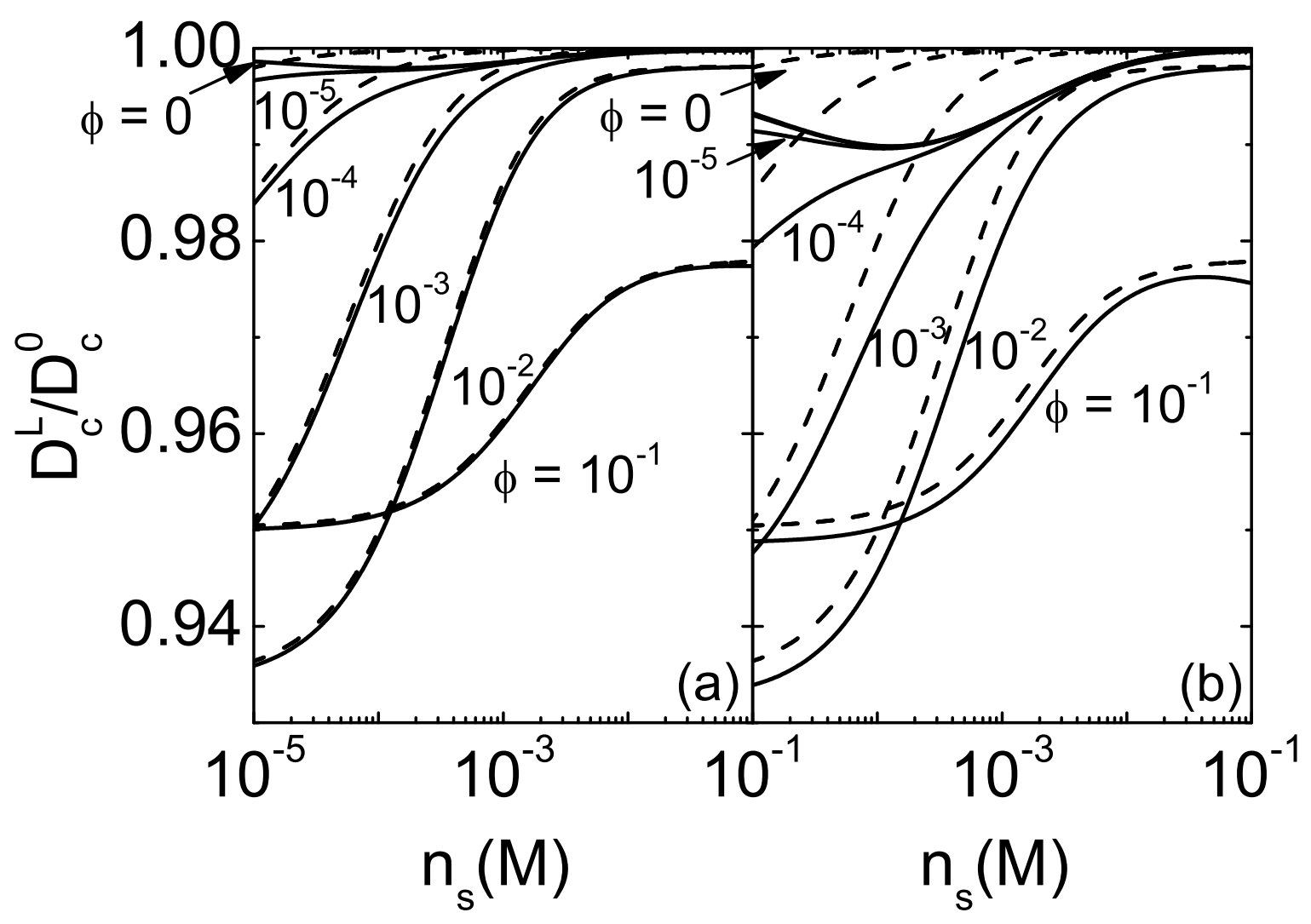


FIG. 3:

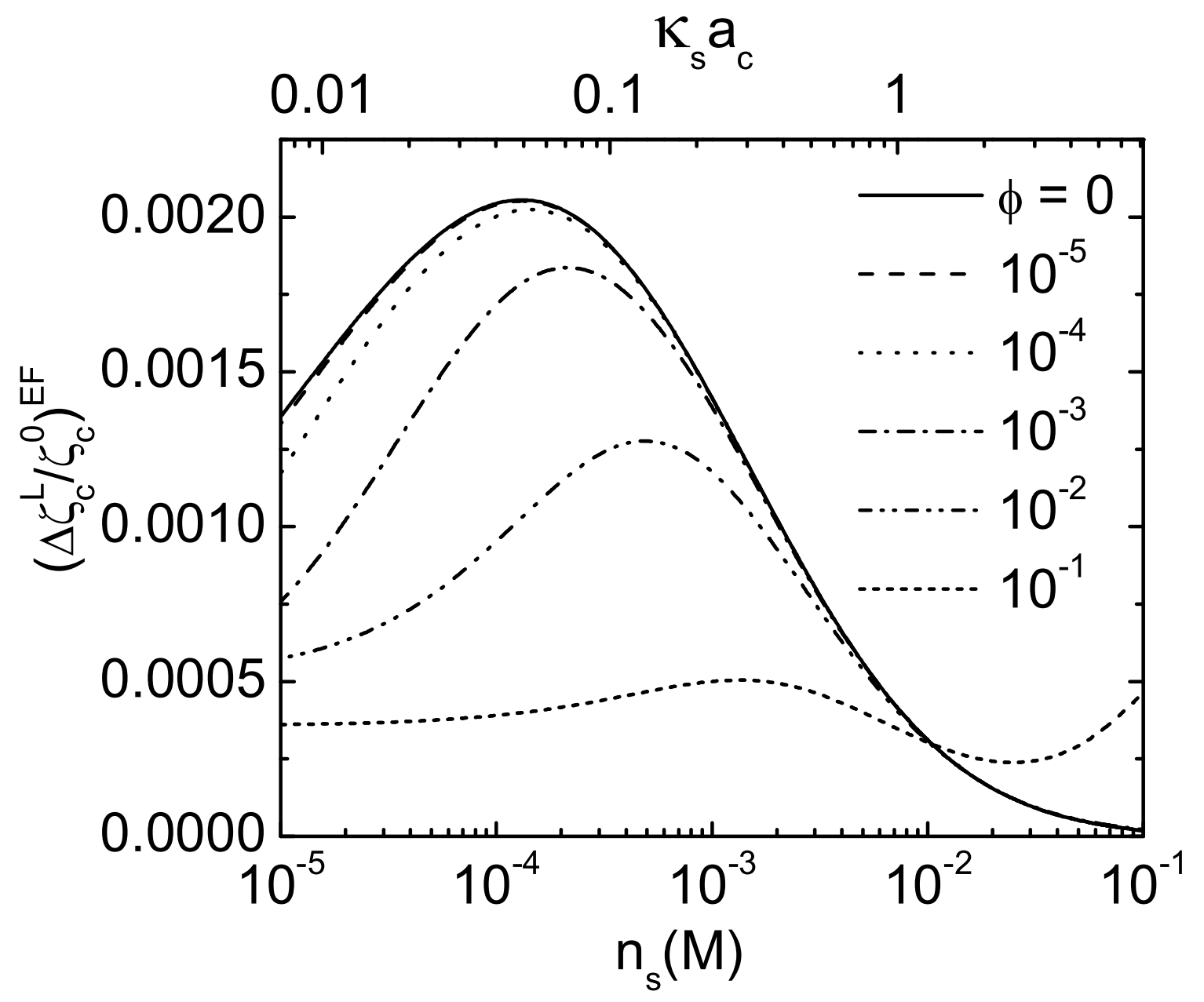


FIG. 4:

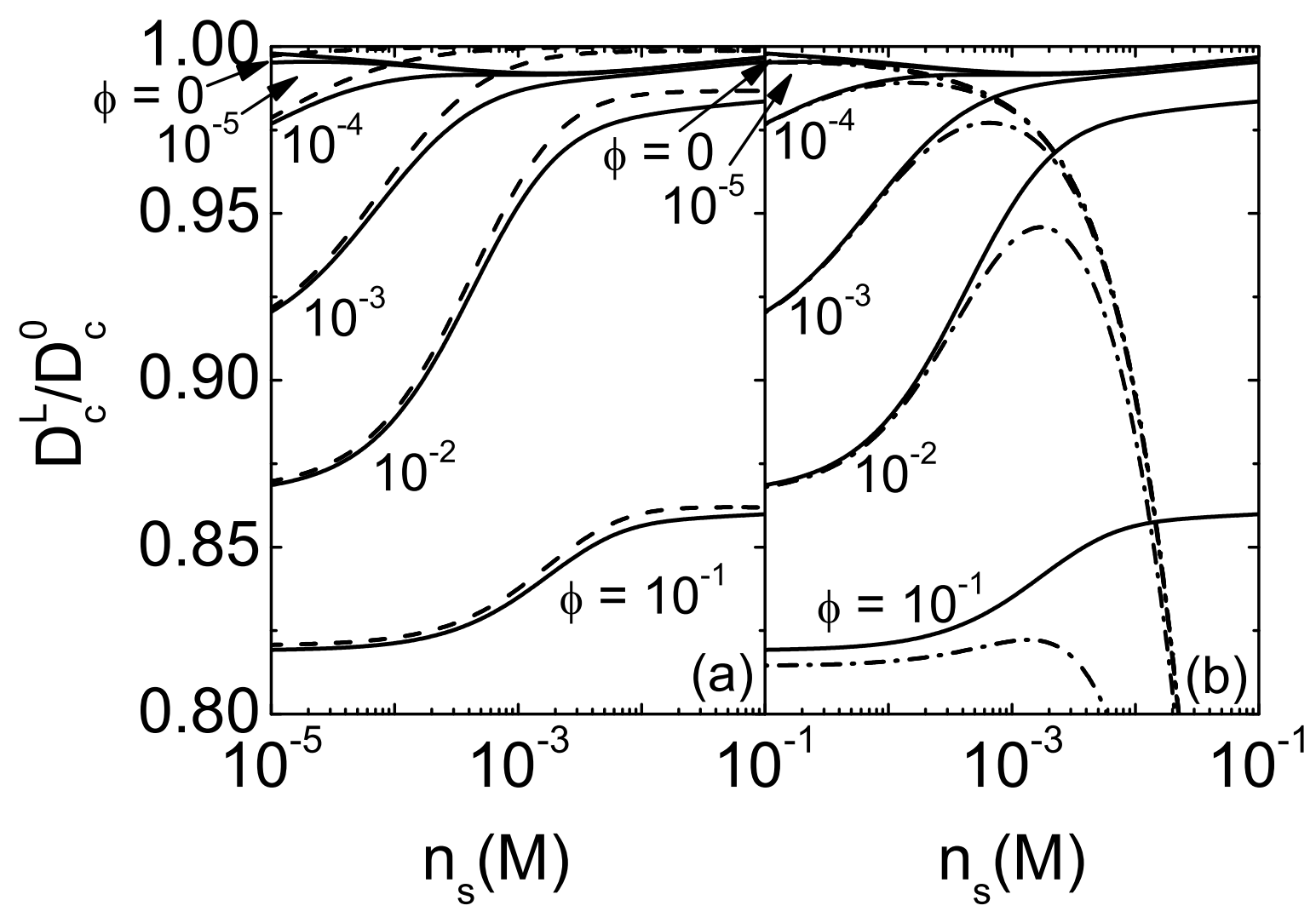


FIG. 5:

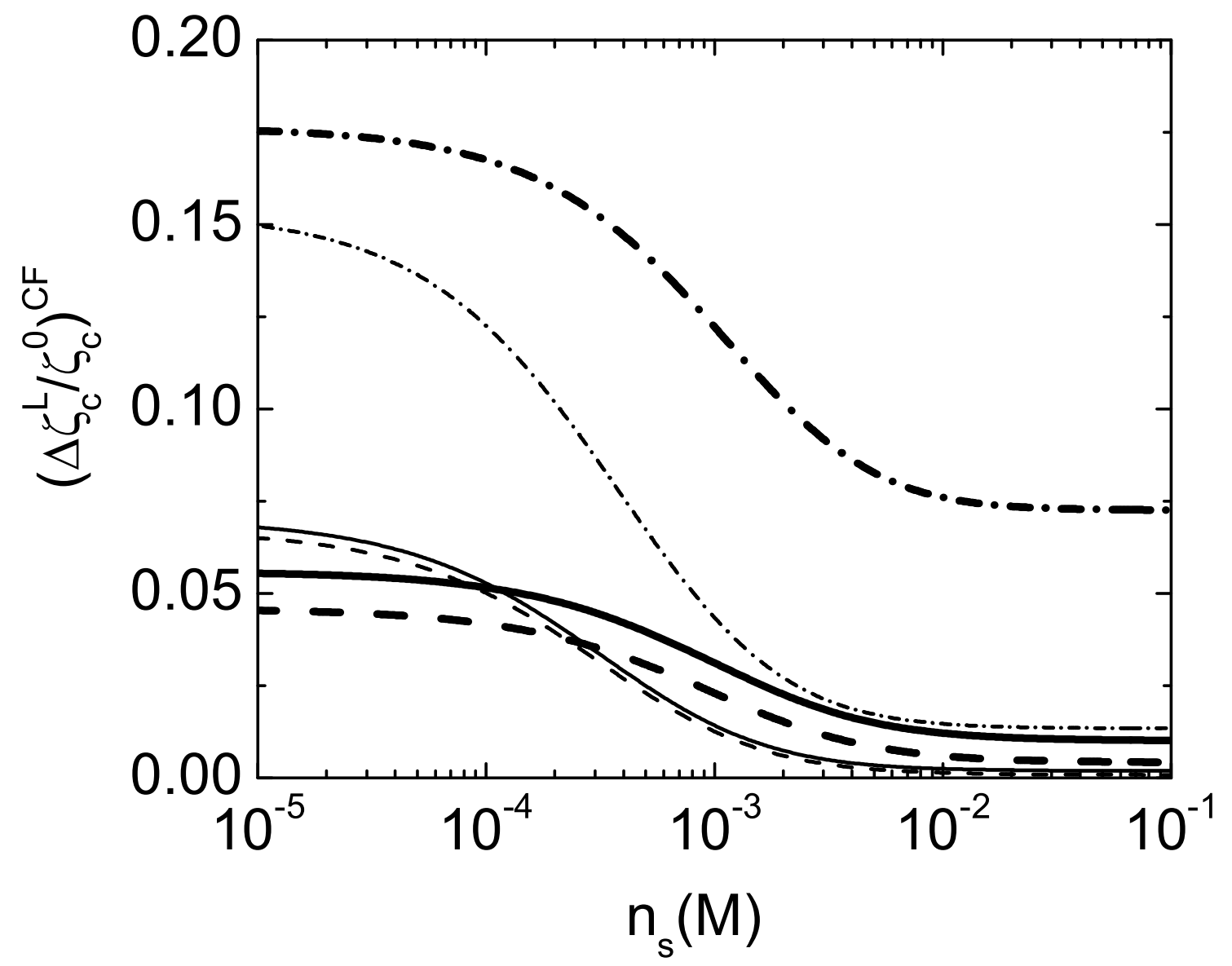


FIG. 6:

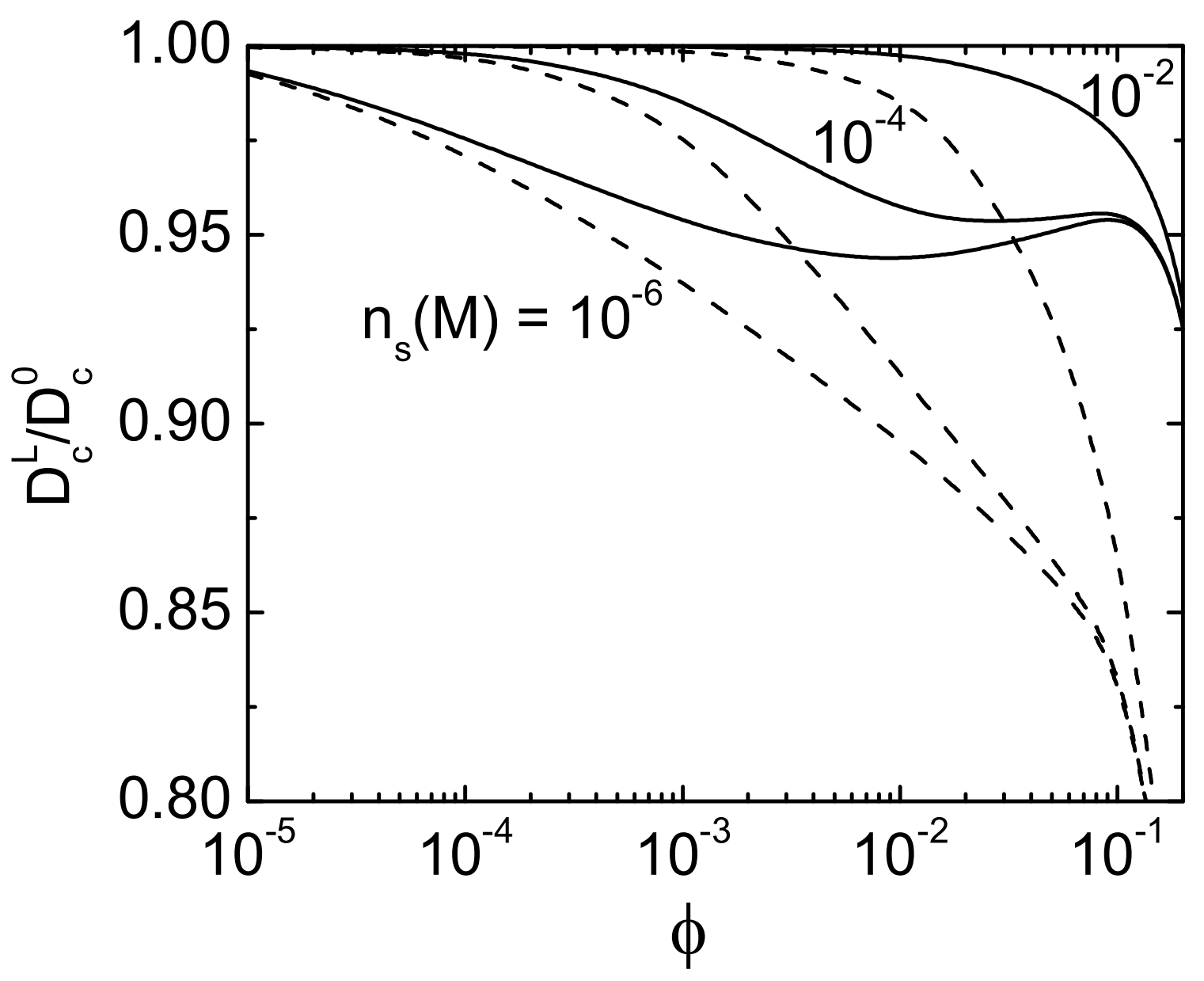


FIG. 7:

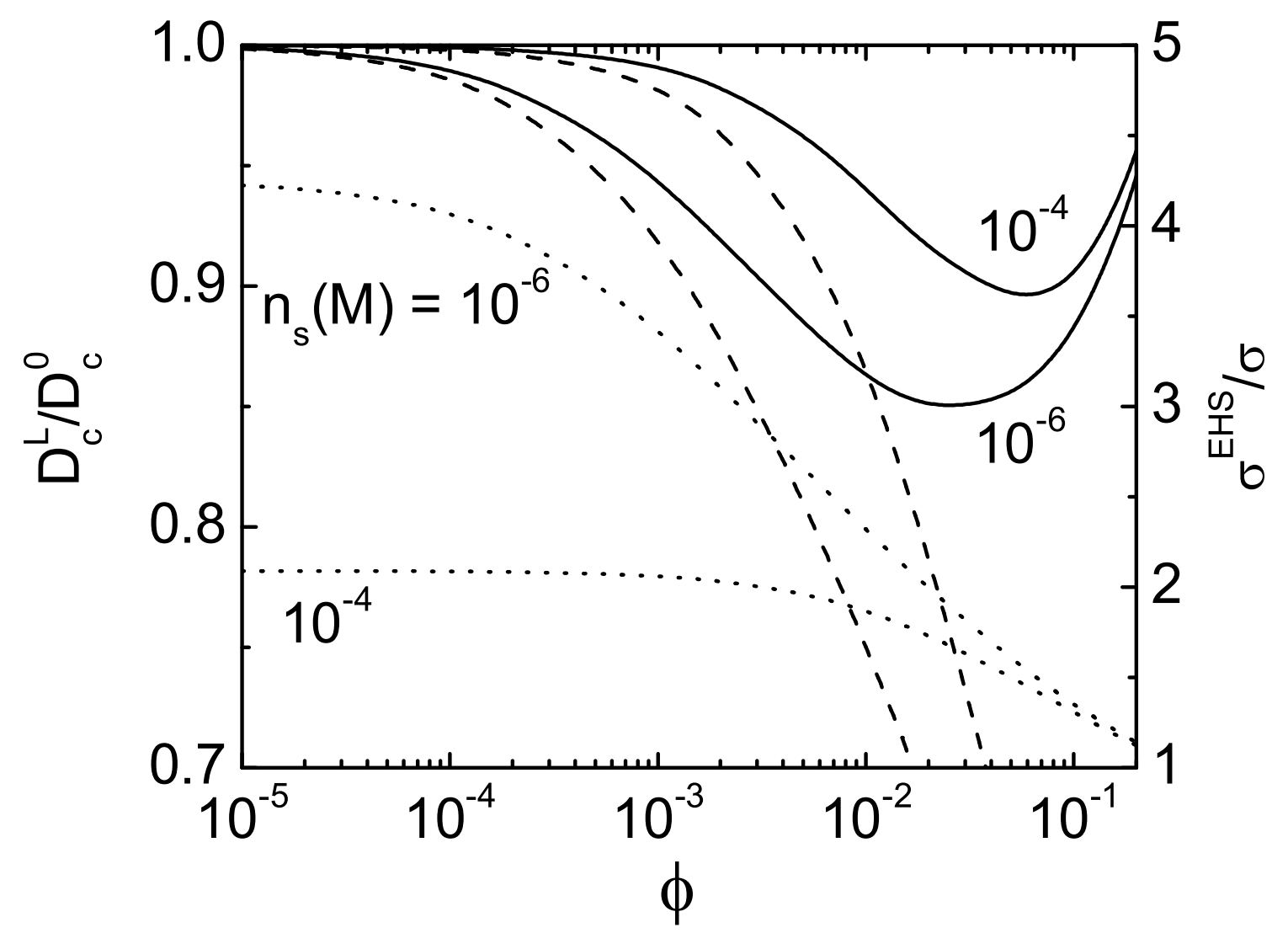

